\title{
La dedicación a los estudios de los jóvenes de origen inmigrante en España en la Gran Recesión

\author{
Immigrant-origin Youth Engagement in Education \\ in Spain during the Great Recession
}

María Miyar-Busto

\author{
Palabras clave \\ Abandono escolar \\ - Aculturación \\ - España \\ - Generaciones 1.5 \\ - Hipótesis del \\ optimismo del \\ inmigrante \\ - Segunda generación \\ de inmigrantes
}

\begin{abstract}
Resumen
En este trabajo se analizan las pautas de dedicación a los estudios de la población joven de origen inmigrante residente en España, a partir de datos de la Encuesta de Población Activa. Los resultados muestran que la probabilidad de estar fuera del sistema educativo de los jóvenes de origen inmigrante es superior a la de los de origen autóctono, aunque solo para los que llegaron a España después de los diez años. Además, su reacción ante cambios en las circunstancias laborales ha sido menos intensa. Asimismo, se encuentra que los recursos educativos paternos frenan la reacción ante cambios en las oportunidades laborales para los hijos de autóctonos y las mujeres hijas de inmigrantes, disminuyendo la desigualdad dentro de cada grupo en contextos de recesión, pero no sucede así para los varones de origen inmigrante.
\end{abstract}

\footnotetext{
Abstract

This paper analyses the patterns of engagement in education of young people of immigrant origin resident in Spain, using data from the Spanish Labour Force Survey. The results show that the probability that immigrant-origin youth may be outside the educational system is higher than the probability for native youth, but only for those who came to Spain after the age of ten years old. In addition, their reaction to changes in the employment situation has been less intense. Also, parental educational resources slow down the reaction to changes in employment opportunities for the children of native people and daughters of immigrants, decreasing the inequality within each group in contexts of recession, but this is not the case for male young immigrants.
}

\section{Cómo citar}

Miyar-Busto, María (2017). «La dedicación a los estudios de los jóvenes de origen inmigrante en España en la Gran Recesión». Revista Española de Investigaciones Sociológicas, 157: 123-140. (http://dx.doi.org/10.5477/cis/reis.157.123)

La versión en inglés de este artículo puede consultarse en http://reis.cis.es

María Miyar-Busto: Departamento de Sociología II (Estructura Social), Universidad Nacional de Educación a Distancia (UNED) | mmiyar@poli.uned.es 


\section{INTRODUCCIÓN ${ }^{1}$}

La sociología de la educación ha encontrado abundante sustento teórico y empírico para validar la relación entre la marcha del mercado de trabajo y la desigual evolución de la dedicación a los estudios entre jóvenes de distinto origen social. Por su parte, la literatura sobre la asimilación de los inmigrantes ha analizado los distintos patrones de dedicación a los estudios de hijos de inmigrantes y autóctonos. Este artículo propone un punto de encuentro entre las dos literaturas, analizando el comportamiento respecto del abandono escolar de los dos colectivos ante cambios en el contexto laboral para el caso español.

El estudio de la dedicación a los estudios de las segundas generaciones de inmigrantes no solo ofrece información relevante sobre la marcha del proceso de asimilación en el país de destino, sino que también resulta muy relevante para predecir las pautas futuras de desigualdad económica y social. Además de las consecuencias que tiene el abandono escolar sobre la evolución del mercado de trabajo, al incorporar a la oferta de trabajo a población sin formación específica (Scarpetta et al., 2010) tiene efectos sobre el bienestar social, la salud y la participación ciudadana (Heckman y LaFontaine, 2010).

Este artículo se propone aportar tres contribuciones principales a la literatura sobre abandono escolar. En primer lugar, una contribución de carácter teórico, en la medida en que pone en relación las teorías de estratificación social sobre las dinámicas del origen social en la determinación de resultados educativos con la literatura sobre la asimilación de las segundas generaciones de inmigrantes. En segundo lugar, pretende contribuir a la mejora del conocimiento empírico sobre la integra-

\footnotetext{
1 Este trabajo ha sido realizado en el marco del proyecto de $\mathrm{I}+\mathrm{D}+\mathrm{i}$ «Reducir el paro estructural en España: formación y empleo, costes laborales, migraciones, Estado de bienestar y familia» (CSO2014-59927-R), financiado por el Ministerio de Economía y Competitividad.
}

ción de los hijos de los inmigrantes en España, a través del análisis de un elemento clave en su futura integración social. En este sentido, el análisis sobre la dedicación a los estudios ofrece información sobre los procesos de asimilación y de perpetuación de la desigualdad entre inmigrantes y autóctonos a través de generaciones. En tercer lugar, intenta proporcionar una mayor comprensión del efecto a largo plazo de los cambios en el ciclo económico para las segundas generaciones de inmigrantes. Para ello, utilizaré datos de la Encuesta de Población Activa (EPA) correspondientes al periodo 2007-2014, analizando las pautas de no dedicación a los estudios de los hijos de inmigrantes y los hijos de autóctonos.

Los objetivos de este artículo son dos. En primer lugar, explorar cómo ha condicionado el origen inmigrante el posible cambio de pautas de dedicación a los estudios con el cambio de ciclo económico. Por último, se propone explorar el distinto papel que juegan los recursos educativos paternos para los jóvenes de origen inmigrante y los hijos de autóctonos en ese cambio de dinámica respecto los estudios.

Existen abundantes contribuciones sobre los efectos que una recesión económica puede tener sobre distintos aspectos de la desigualdad entre autóctonos e inmigrantes, como los resultados en el mercado laboral (Muñoz-Comet, 2013; Garrido et al., 2010) o el retorno de la educación (Cebolla-Boado et al. , 2015). Sin embargo, hasta ahora no se ha analizado el efecto que una recesión puede tener sobre la desigualdad en la probabilidad de estar fuera del sistema educativo según el origen inmigrante o no.

Con este propósito, el trabajo seguirá el siguiente orden. Primero, se describirá la evolución de la población residente en España y la marcha del mercado de trabajo en relación con las pautas de no dedicación a los estudios. En segundo lugar, se expondrán las principales líneas teóricas en el análisis del abandono escolar, así como las hi- 
pótesis del estudio. Tercero, se detallarán los datos, variables y metodología que se empleará en la parte empírica del análisis. En cuarto lugar, se presentarán los resultados descriptivos y de los análisis multivariantes, y, por último, se expondrán las conclusiones.

\section{LOS JÓVENES EN LA INTERSECCIÓN DEL SISTEMA EDUCATIVO, MERCADO LABORAL Y CICLO ECONÓMICO EN ESPAÑA}

A finales de los años setenta, España había emprendido una senda de disminución del abandono escolar que se detuvo a mediados de los noventa debido a factores como la creciente disponibilidad de puestos de trabajo poco cualificados o el cambio en el diseño del itinerario educativo (Felgueroso et al., 2014). Esta tendencia contrasta con el patrón de disminución del abandono escolar en el conjunto de la Unión Europea de los 15. Según datos de Eurostat a partir de las Encuestas de Población Activa europeas, en un año de pleno auge económico, como 2005, el abandono escolar en España se situaba en el $37 \%$ de los hombres y el $25 \%$ de las mujeres, frente al 20 y el $15 \%$ respectivamente para el conjunto de la Unión Europea de los 15.

A partir de 2008 la intensa recesión sufrida por la economía española supuso la destrucción de más de tres millones de empleos en siete años. La literatura ha puesto de relieve que el aumento del desempleo afectó especialmente a los jóvenes con poca cualificación (Dolado et al., 2013), al mismo tiempo que a los sectores y ocupaciones en los que se habían insertado la mayoría de los inmigrantes durante la expansión (Bernardi et al., 2011). Asimismo, se constata un incremento de la relevancia del nivel educativo en la determinación de la situación laboral durante la recesión (Cebolla-Boado et al., 2015).

Desde 2008, según los datos de Eurostat, el abandono escolar en España se reduce, sobre todo para los hombres, aunque sigue siendo superior al de las mujeres, de modo que en 2014 afectaba al 26\% de los hombres, frente al $18 \%$ de las mujeres. A pesar de la notable mejora, las magnitudes siguen siendo considerablemente mayores que las europeas, que durante el mismo periodo también continuaron disminuyendo, de modo que en la misma fecha se correspondían con el 13\% de los hombres y el $10 \%$ de las mujeres.

Por otro lado, tanto durante la expansión económica como tras el cambio de ciclo, los inmigrantes residentes en España han emprendido proyectos de formación familiar, bien a través de la reunificación como de la formación de nuevos núcleos en destino (Castro Martín y Rosero-Bixby, 2011; Requena y Sánchez-Domínguez, 2011). Según datos de la Estadística de Variaciones Residenciales, el porcentaje de menores de 16 años sobre el total de entradas de población nacida en el extranjero se ha mantenido alrededor del 15\% desde 2000 hasta 2014. Además, el porcentaje de nacimientos de madre extranjera superó en 2008 el $20 \%$ del total de nacimientos. Un indicador de la intensidad del proceso de formación familiar de los nuevos residentes es el espectacular aumento de la población empadronada extranjera menor de 16 años residente en España, que ha pasado de 133.391 en 2000 a 798.601 en 2014. Se trata por lo tanto de un grupo de población creciente y notable en su magnitud, del que la literatura sobre la asimilación de las segundas generaciones de inmigrantes (o generaciones 1.5) espera patrones diferenciados respecto a su inclusión en el sistema educativo una vez finalizada la edad de escolarización obligatoria.

\section{LOS DETERMINANTES DE LA DEDICACIÓN A LOS ESTUDIOS PARA LOS HIJOS DE AUTÓCTONOS Y LOS HIJOS DE INMIGRANTES}

La literatura que ha tratado de explicar los determinantes de la dedicación a los estudios de los inmigrantes ante cambios en el 
contexto económico parte de dos líneas de análisis. La primera se refiere a las teorías de la estratificación educativa que analizan los determinantes del logro educativo de distintos grupos de población, y la segunda línea de investigación pone el énfasis en las variaciones de la dedicación a los estudios ante cambios en el contexto económico.

Las teorías sobre la estratificación educativa formulan una distinción clásica que hace referencia a la existencia de dos procesos que explican el logro educativo. Por un lado, los resultados educativos o habilidades cognitivas, o efectos primarios, y por otro lado, las elecciones educativas, que se realizan en función de una estructura de beneficios y costes, también llamados efectos secundarios (Boudon, 1974).

Varios trabajos recientes desde la sociología de la educación han aportado evidencias empíricas sobre la desventaja que tienen los inmigrantes respecto a los autóctonos en los efectos primarios, al mismo tiempo que gozan de una ventaja en los efectos secundarios, para los casos de Suecia (Jonsson y Rudolphi, 2011), Francia (Cebolla-Boado, 2011) y Navarra (Cebolla-Boado y Martínez de Lizarrondo, 2015). Este argumento estaría en consonancia con la hipótesis del optimismo de los inmigrantes (Kao y Tienda, 1995), que predice una mayor dedicación a los estudios de los hijos de inmigrantes, una vez controladas las desventajas a las que se enfrentan, como un peor dominio del idioma o una peor posición de clase. Esta mayor dedicación neta a los estudios se explicaría por un mayor énfasis de las familias inmigrantes en la importancia del logro educativo y los valores familiares. Existe abundante literatura que ha intentado validar esta hipótesis, sobre todo para el caso norteamericano (Driscoll, 1999; Harris et al., 2008; Landale et al., 1998; Perreira et al., 2006; Pong et al., 2005; Rumbaut, 1997; White y Glick, 2009).

En contraposición a este tipo de análisis, los estudios que se han centrado en la asi- milación de las segundas generaciones de inmigrantes han encontrado una desventaja neta en su logro educativo. Esta desventaja se explicaría a partir de la hipótesis de la asimilación segmentada, que plantea que los inmigrantes se insertan en distintas pautas de movilidad social en función de los recursos disponibles a su llegada, de modo que mientras que unos pueden seguir pautas de movilidad social ascendente, otros no podrán seguir esa senda. Un último grupo emprenderá una asimilación limitada, con aspiraciones educativas para sus hijos pero restringiendo su integración en la cultura de acogida. Desde esta línea de análisis se ha prestado especial atención al concepto de aculturización que pueden sufrir las segundas generaciones de inmigrantes (Greenman, 2013). Desde esta perspectiva, la hipótesis de la asimilación segmentada implica que las segundas generaciones de inmigrantes que no emprenden la pauta de movilidad social ascendente tendrán unas aspiraciones educativas más bajas (Portes y Zhou, 1993). La literatura ha encontrado evidencias contrapuestas. Mientras que Hirschman (2001) encuentra evidencia empírica de la existencia de aculturación y asimilación segmentada con datos para Estados Unidos, también descubre que su existencia depende en gran medida del origen y de la edad en que llegaron a Estados Unidos. Haller et al. (2011) también encuentran un patrón de asimilación segmentada y movilidad descendente. No obstante, Farley y Alba (2002), Waldinger y Feliciano (2004) y Xie y Greenman (2011) no encuentran evidencias de que la segunda generación de inmigrantes experimente movilidad descendente.

Por otra parte, la segunda línea de análisis relevante para este trabajo se ocupa del análisis de la desigual repercusión que un cambio de ciclo económico tiene en el abandono escolar en función del origen social, en la medida en que modifica la estructura de costes y beneficios de continuar estudiando de forma distinta a diferentes grupos de población. 
Ante la llegada de un shock externo como una recesión económica, la literatura se plantea dos posibles mecanismos con consecuencias opuestas sobre la continuidad de los estudiantes de distintas clases sociales en el sistema educativo. Por una parte, podría darse un efecto sustitución, según el cual la reducción de oportunidades en el mercado laboral, en la medida en que se hubieran destruido en mayor medida ocupaciones de nivel bajo, disminuirían el coste de oportunidad de seguir estudiando y por lo tanto aumentará la probabilidad de que los jóvenes con orígenes sociales más desfavorecidos permanecieran en el sistema educativo. Por otra parte, también se plantea la posibilidad de que opere un efecto renta, en base al cual la disminución del presupuesto de los hogares, el aumento de los costes educativos o la disminución de la calidad de la educación redujeran la dedicación a los estudios de las posiciones sociales más bajas en mayor medida que la de las más altas (Cebolla-Boado et al., 2015).

En base a estas líneas de análisis, se deducen tres posibles fuentes de diferenciación de las pautas de los jóvenes de origen inmigrante en sus patrones de abandono escolar en un contexto de cambio económico:

A) Los inmigrantes pueden tener distintos patrones de abandono escolar, bien porque estén inmersos en procesos de aculturización, planteados en la tesis de la asimilación segmentada, o bien en razón de las mayores aspiraciones de movilidad social planteadas en la hipótesis del optimismo de los inmigrantes. Nuevamente, el sentido en el que opere el componente de origen inmigrante dependerá de cuál de los dos elementos funcione con mayor intensidad sobre las aspiraciones de la segunda generación y sus familias. Este componente de origen inmigrante puede tener consecuencias tanto sobre la probabilidad de no dedicarse a los estudios como sobre la capacidad de reacción ante cambios en el ciclo económico.
B) El deterioro de las oportunidades laborales suele ser más intenso entre las peores posiciones en el mercado de trabajo y estas a su vez suelen estar ocupadas por los inmigrantes (Farkas, 1996; Zhou, 1997), especialmente en los países del sur de Europa (Causa y Jean, 2007; Kogan, 2006). Se trataría por lo tanto de un componente de origen social. El sentido en el que interviene este componente dependerá de los pesos relativos del efecto renta y el efecto sustitución.

C) El componente de origen social puede operar de forma distinta entre los hijos de inmigrantes que entre los hijos de autóctonos. Las familias inmigrantes que se sitúan en las posiciones menos acomodadas son más susceptibles de carecer del apoyo público o las redes sociales necesarias para evitar tener que incorporar a otro miembro de la familia al mercado laboral. Alternativamente, la posible mayor valoración del logro educativo en estas familias puede repercutir en una reacción más positiva que la de las familias nativas de igual origen social. El componente de origen inmigrante operaría aquí como un mediador del componente de origen social.

\section{Hipótesis}

En definitiva, este trabajo se plantea testar las siguientes dos hipótesis y sus alternativas, en base a dos preguntas de investigación:

1) ¿Los hijos de los inmigrantes reaccionan más intensamente ante cambios en el contexto económico en su probabilidad de no cursar estudios reglados?

H1a: Los hijos de los inmigrantes aumentan más su dedicación a los estudios que los hijos de autóctonos en un periodo de recesión económica.

H1b: Los hijos de los inmigrantes aumentan menos su dedicación a los estudios que los 
hijos de autóctonos en un periodo de recesión económica.

2) ¿Funciona el origen inmigrante como un mediador del efecto del origen social ante cambios en el contexto económico?

H2a: El componente de origen inmigrante opera como un mediador que mejora en términos relativos la reacción de los hijos de los inmigrantes con menos recursos educativos en contextos de cambio económico.

H2b: El componente de origen inmigrante opera como un mediador que empeora en términos relativos la reacción de los hijos de los inmigrantes con menos recursos educativos en contextos de cambio económico.

\section{Datos, VARIABLes Y MÉTOdOS}

En este artículo se utilizarán los microdatos de la EPA española. Esta encuesta ofrece información sobre la evolución del mercado de trabajo y las características de los hogares. Es una encuesta trimestral que se realiza en España desde 1964 y cuenta con una muestra de alrededor de 60.000 hogares, sobre 180.000 personas. En este análisis se usan datos de las encuestas comprendidas entre 2007 y 2014. Este periodo comprende así desde el punto de partida de la recesión (2007 fue el último año del periodo expansivo en que la tasa de empleo subió) hasta el año en que la tasa de empleo volvió a crecer. Esta elección temporal nos permite además contar con la información del año de llegada de todos los jóvenes nacidos en el extranjero?

La población objeto de análisis será la comprendida entre los 16 (edad a la que deja de ser obligatorio estudiar) y los 19 , ambos

\footnotetext{
2 Hasta 2007 la EPA solo incluía la información de años de residencia en el país para las personas sin nacionalidad española. Desde 2007 también figura esta variable para las personas con nacionalidad española que han nacido fuera.
}

incluidos. Con el propósito de poder comprender información sobre el origen social, en el análisis se restringe el grupo analizado a aquellos que viven en el mismo hogar que al menos uno de sus padres. En el caso del grupo de edad de 16 a 19 años, según datos de la EPA, el grupo excluido es minoritario. La muestra total de este grupo para el periodo estudiado es de 183.985 jóvenes, de los que 17.191 tienen padres nacidos en el extranjero.

En cuanto a la variable dependiente de la investigación, se trata de no cursar estudios reglados. Entre las variables independientes principales se incluirá el origen inmigrante del joven, especificado como la situación en la que los dos padres hayan nacido fuera de España (o uno de ellos en el caso de que solo convivan con uno). Asimismo, los jóvenes inmigrantes se clasificarán en función de su nacimiento en España y, en caso contrario, de si han llegado antes o después de haber cumplido los 10 años. De esta forma se tendrá en cuenta el tiempo de residencia, que se espera que sea una variable clave en la integración social de los jóvenes. Para facilitar la simplicidad del análisis, se excluyen a los hijos de parejas mixtas, cuyos resultados resultan muy similares a los de los hijos de autóctonos ${ }^{3}$.

Además, se incluirá en las estimaciones información sobre los recursos educativos paternos (se tomará el nivel educativo más elevado entre el de los dos progenitores). El nivel educativo de los padres está clasificado en Secundaria Inferior o menos, Formación Profesional I, Secundaria Superior, Formación Profesional II y Estudios Universitarios. Para parte del análisis descriptivo, así como para los análisis en los que se incluye una interacción entre la evolución del mercado de trabajo y el nivel educativo paterno, se agregará esta última variable en una variable dicotómica en la que los hijos de inmigrantes con es-

3 Estimaciones disponibles bajo demanda. 
tudios de Formación Profesional Superior o Estudios Universitarios toman valor uno. Asimismo, se incluirá en el análisis una variable relativa a los recursos materiales del hogar, especificada en función de la situación laboral de los progenitores: si ninguno de los dos está empleado, solo uno de ellos, o los dos.

Para especificar en las estimaciones el efecto de la evolución del mercado de trabajo, se incluye una variable independiente que consiste en el cambio porcentual en el porcentaje de ocupados respecto del total del grupo de edad (de 16 a 19 años) de cada sexo y origen migratorio. Se trata de capturar así la variación en la probabilidad real que tendrían los jóvenes de cada grupo demográfico considerado de encontrar un empleo.

Para las estimaciones restringidas a la población de origen inmigrante, se incluyen también algunas variables específicas del colectivo que se espera que tengan influencia sobre su dedicación a los estudios: región de origen de los padres (UE-15, resto de Europa, Latinoamérica, África, Asia y otros países occidentales) y disfrutar o no de la nacionalidad española.

Dadas las distintas pautas de hombres y mujeres, tanto en cuanto a su comportamiento laboral como en cuanto a su dedicación a los estudios, los análisis se realizarán de forma separada para hombres y mujeres. Se controlará también el análisis por la variable edad.

La metodología a seguir para el análisis empírico constará de dos pasos. En primer lugar, se realizará un análisis descriptivo en el que se presente la pauta temporal de no dedicación a los estudios de los jóvenes en función de su estatus migratorio, el sexo y los recursos educativos paternos. En segundo lugar se estimarán una serie de modelos de regresión logística. En el primer bloque de modelos se estimará la probabilidad de no cursar estudios reglados para todos los jóvenes, incluyendo la interacción entre la variación en la tasa de ocupación y el origen mi- gratorio de los jóvenes. En el segundo bloque de modelos se realizarán estimaciones por separado para los jóvenes de origen autóctono y los jóvenes de origen inmigrante, incluyendo una interacción entre la variación en la tasa de ocupación y el nivel educativo de los padres. Las estimaciones correspondientes a los jóvenes de origen inmigrante se completarán, asimismo, con las variables específicas al colectivo que pueden influir en su proceso de integración social.

En los cuadros se presentarán los efectos marginales medios, que permiten la comparación de coeficientes entre modelos estimados para distintas muestras y con distintas variables (Mood, 2010), los errores estándar y los niveles de significatividad.

\section{Resultados}

\section{Análisis descriptivo}

A la luz de la información recogida en el gráfico 1, durante todo el periodo analizado el porcentaje de jóvenes que no realizan estudios reglados es superior entre los hijos de los inmigrantes que entre los hijos de autóctonos, así como menor entre las mujeres de los dos grupos. En 2007, casi un tercio de los hombres de origen autóctono y un quinto de las mujeres no estaban estudiando, frente a un $48 \%$ de los varones jóvenes de origen inmigrante y un $38 \%$ de las mujeres.

Sin embargo, la distinta evolución de la presencia en el sistema educativo de los cuatro colectivos hace que la distancia no sea constante a lo largo del periodo. Por una parte, tanto entre los hombres como entre las mujeres jóvenes de origen español se aprecia una disminución suave de los porcentajes de no estudiantes desde 2007 y que se acelera a partir de 2008. Por otra parte, entre los hombres jóvenes de origen inmigrante la reducción del porcentaje de jóvenes que no estudian no se acelera hasta 2009, aunque entre las mujeres del mismo colectivo la reducción sí se observa ya desde 2007. 
Asimismo, a partir de 2009 la contracción en el porcentaje de jóvenes fuera del sistema educativo es más rápida entre los hijos de inmigrantes que entre los hijos de autóctonos, de modo que la distancia entre los dos grupos disminuye hasta alcanzar un mínimo en 2012. A partir de ese año, mientras que los jóvenes de origen nativo continúan la pauta precedente de disminución del porcentaje de jóvenes que no se dedican a los estudios, los de origen inmigrante la frenan e incluso la aumentan ligeramente. En 2014 el 15\% de los hombres jóvenes de origen autóctono no realizaba estudios reglados, frente al $24 \%$ de los de origen inmigrante. Entre las mujeres, las cifras correspondientes eran del 10 y del $19 \%$ respectivamente.

En los gráficos 2 y 3 se presenta la evolución del porcentaje de jóvenes no estudiantes de los cuatro colectivos de forma separada para los hijos de padres con nivel educativo sin y con educación superior (educación universitaria o Formación Profesional superior). De la información recogida en los gráficos se deducen tres dinámicas. En primer lugar, el porcentaje de jóvenes que no cursan estudios reglados es menor entre los hijos de padres con mayor nivel educativo para todos los colectivos considerados en función del origen inmigrante y el sexo. La media del periodo para los hombres y mujeres jóvenes de origen autóctono y padres sin estudios superiores es del 28 y del $19 \%$ respectivamente, mientras que para los hijos e hijas de padres con mayor nivel educativo es del 9 y del 6 respectivamente. Entre los jóvenes de origen inmigrante, los porcentajes se corresponden con el 38 y el $31 \%$ para los hijos e hijas de padres sin estudios superiores frente al 23 y al $17 \%$ para los jóvenes con padres con estudios superiores.

GRÁFICO 1. Porcentaje de jóvenes que no cursan estudios reglados, por sexo y origen autóctono o inmigrante, 16-19 años

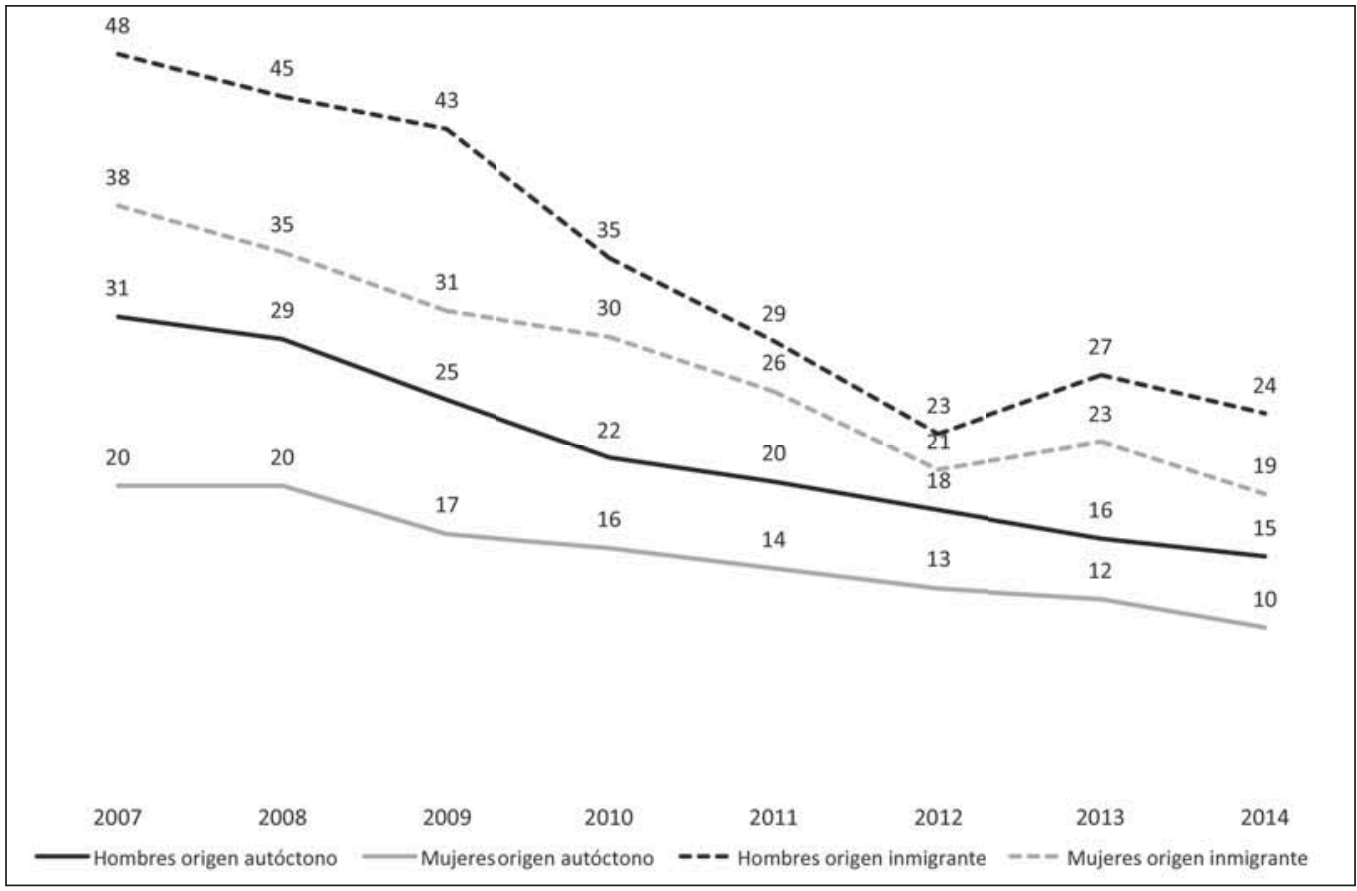

Fuente: Elaboración propia a partir de EPA 2007-2014. 
GRÁFICO 2. Porcentaje de jóvenes que no cursan estudios reglados, por sexo y origen autóctono o inmigrante, 16-19 años, padres sin estudios superiores

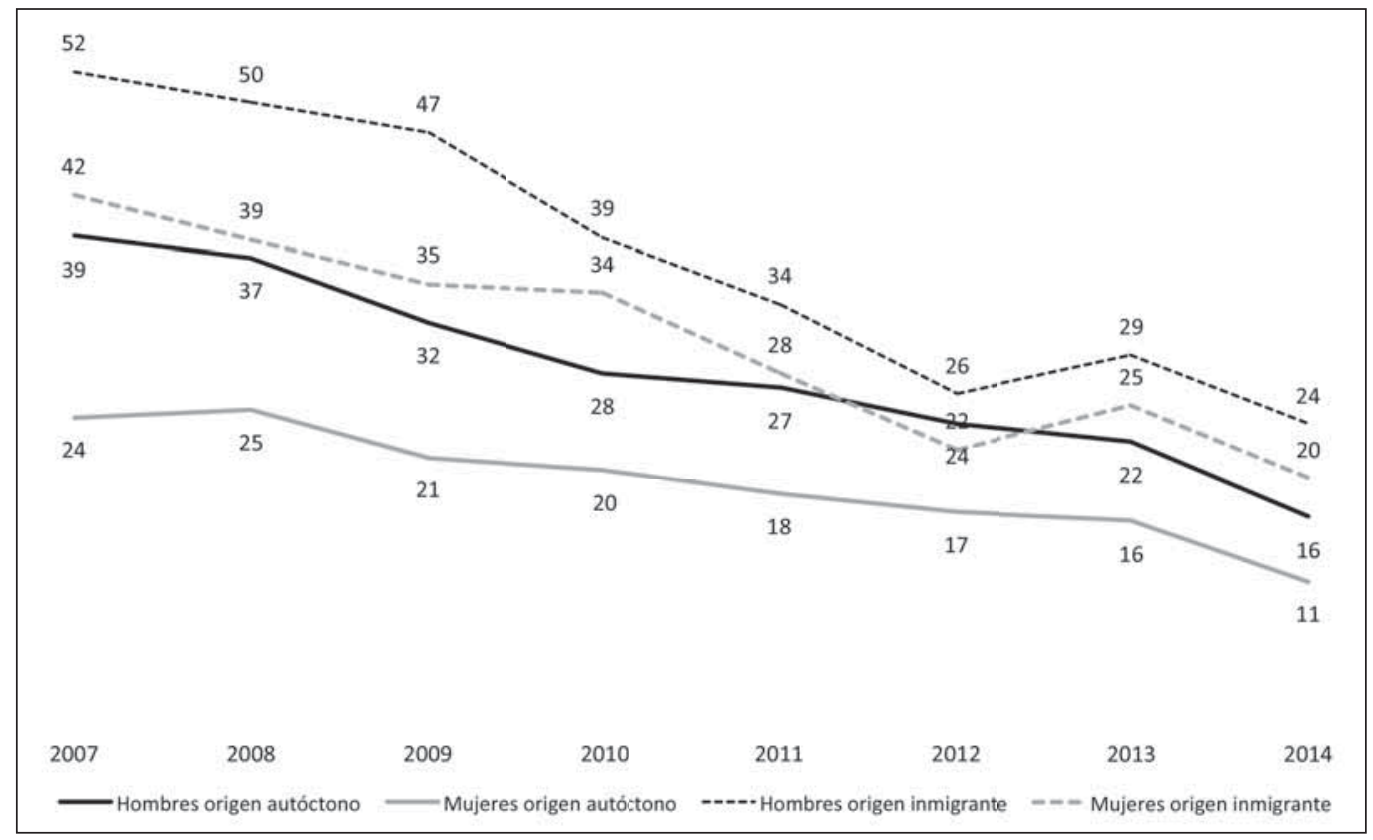

Fuente: Elaboración propia a partir de EPA 2007-2014.

GRÁFICO 3. Porcentaje de jóvenes que no cursan estudios reglados, por sexo y origen autóctono o inmigrante, 16-19 años, padres con estudios superiores

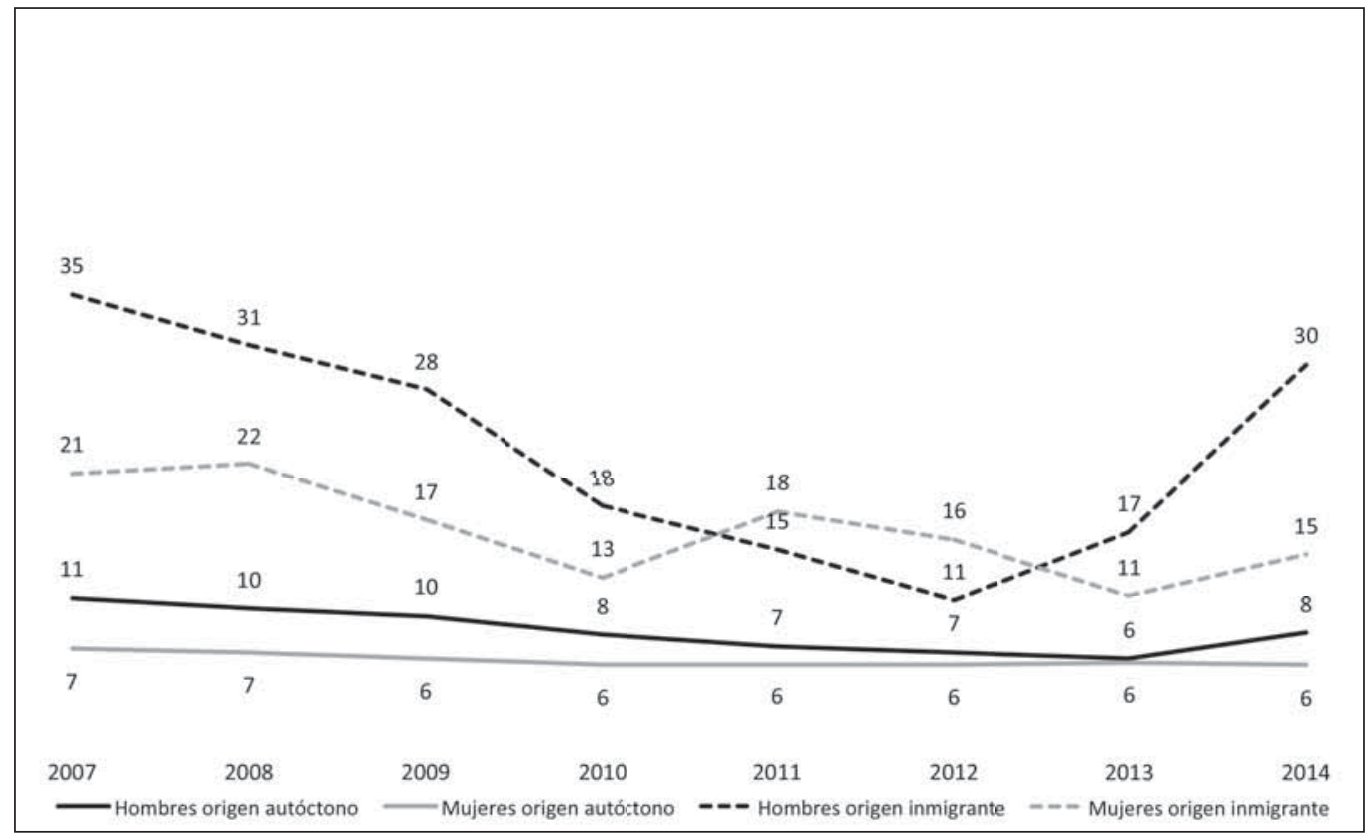

Fuente: Elaboración propia a partir de EPA 2007-2014. 
En segundo lugar, cabe señalar que la distancia en función del estatus migratorio es mayor entre los jóvenes con padres con más recursos educativos que entre los hijos de padres con menos estudios. Mientras que entre los jóvenes con padres sin estudios superiores la diferencia que supone ser de origen inmigrante en el porcentaje de jóvenes que no estudian es de un 33 y un $60 \%$, entre los jóvenes de padres con mayor educativo esta distancia es del $160 \%$, tanto en el caso de los hombres como de las mujeres.

Por último, a pesar de que los dos grupos por nivel educativo evolucionan en el tiempo de forma similar en cuanto a la distancia entre jóvenes de origen inmigrante y nativo, las magnitudes difieren. Así, mientras que en 2014 la relación entre los porcentajes de jóvenes de origen inmigrante y autóctono que no estudian se mantenía en niveles similares a los previos a la crisis para los hijos con padres de bajo nivel educativo, la distancia había crecido entre los hijos con padres de mayor nivel educativo, fundamentalmente debido a las pautas diferenciales a partir de 2012 .

\section{Modelos multivariantes}

Los resultados de la estimación multivariante confirman que el origen inmigrante condiciona la probabilidad de estar en el sistema educativo en este tramo de edad (cuadro 1). Sin embargo, entre los varones, solo aquellos que llegaron a España después de los diez años tienen una probabilidad mayor que los descendientes de autóctonos de no cursar estudios reglados (un 10\% superior). De hecho, los jóvenes varones nacidos en España con padres inmigrantes tienen una probabilidad un $4 \%$ menor que los de origen autóctono de no estudiar, y los que llegaron antes de los 10 años no muestran ninguna diferencia significativa. Entre las mujeres, aunque las nacidas en España de padres inmigrantes no presentan ninguna diferencia significativa con las hijas de padres autóctonos, las que llegaron a
España antes de los 10 años tienen una probabilidad un 3\% mayor de estar fuera del sistema educativo, y las que llegaron con diez años o más, de un $12 \%$.

Por otra parte, a la luz de los resultados de las estimaciones se deduce que los cambios en la tasa de ocupación tienen un mayor efecto sobre la dedicación a los estudios de los hombres que de las mujeres. Por cada punto porcentual de variación en la tasa de ocupación, la probabilidad de no estudiar aumenta en un 0,6\% para los hombres y un 0,3\% para las mujeres. En 2012, la variación porcentual en la tasa de ocupación de los jóvenes de origen autóctono fue del $-9,1 \%$, lo que implicaría, según las estimaciones, una reducción de la probabilidad de estar fuera del sistema educativo del 5,5\%. Sin embargo, los jóvenes de origen inmigrante ven reducido este efecto en un $0,5 \%$ por punto porcentual, lo que implica que su sensibilidad a cambios en las condiciones del mercado de trabajo es notablemente menor: ante una disminución en la tasa de ocupación, aumentan menos su presencia en el sistema educativo que los jóvenes de origen autóctono. En el caso de las mujeres la interacción de la evolución de la ocupación con el componente inmigrante se reduce a un $0,1 \%$, de modo que se podría afirma que la diferencia en su nivel de reacción respecto a sus pares de origen autóctono es menor que en el caso de los hombres.

La estimación también confirma otras predicciones recurrentes en la literatura sociológica sobre dedicación a los estudios, como la influencia negativa del mayor nivel educativo de los padres en la probabilidad de estar fuera del sistema educativo. Los hijos e hijas de universitarios tienen una probabilidad un 31 y un $21 \%$ menor, respectivamente, de estar fuera del sistema educativo que los hijos de los que tienen estudios de secundaria inferior o menos. El efecto de los recursos educativos de los progenitores es por lo tanto un $50 \%$ mayor para los hombres que para las mujeres jóvenes. Además, la 
relación de la dedicación a los estudios con el nivel educativo paterno es más intensa que con la situación laboral de los padres. Un joven con dos padres ocupados tiene una probabilidad un $8 \%$ menor de no cursar estudios reglados que un joven sin ningún padre ocupado en el hogar.

En el segundo grupo de modelos (cuadro 2) se profundiza en diferentes mecanismos que afectan a la probabilidad de estar fuera del sistema educativo. En esta parte del análisis se han realizado estimaciones por separado en función del origen inmigrante y el sexo en las que se ha incluido una variable de interacción entre el nivel educativo de los padres y la variación en la tasa de ocupación. De esta forma se capturan las posibles diferencias en intensidad y dirección del efecto del origen social ante cambios en las condiciones de empleo.

De los resultados de las estimaciones se desprende, en primer lugar, que los recursos educativos juegan un papel menos relevante en la determinación de la probabilidad de no realizar estudios reglados para los jóvenes de origen inmigrante, especialmente para los hombres. En segundo lugar, a la luz de las estimaciones se deduce que los cambios en el mercado de trabajo no han traído consigo un efecto renta sobre la probabilidad de estar fuera del sistema educativo, puesto que en ninguno de los cuatro casos considerados los jóvenes en hogares con menos recursos educativos reaccionan disminuyendo

CUADRO 1. Logit de la probabilidad de no cursar estudios reglados, efectos marginales medios, por sexo

\begin{tabular}{|c|c|c|c|c|}
\hline & \multicolumn{2}{|c|}{ Hombres } & \multicolumn{2}{|c|}{ Mujeres } \\
\hline & EMM & E.E. & EMM & E.E. \\
\hline \multicolumn{5}{|l|}{ Origen (ref.: hijo de nativos) } \\
\hline Hijo de inmigrantes nacido en España & $-0,04^{\star \star \star}$ & 0,010 & 0,01 & 0,009 \\
\hline Llegada a España antes de los 11 años & $-0,01$ & 0,007 & $0,03^{\star \star \star}$ & 0,006 \\
\hline Llegada a España después de los 10 años & $0,10^{* * *}$ & 0,006 & 0,12 *** & 0,005 \\
\hline Crecimiento del empleo & $0,006 * \star \star$ & 0,000 & $0,003^{* \star *}$ & 0,000 \\
\hline Crecimiento del empleo * origen inmigrante & $-0,005$ *** & 0,000 & $-0,001^{\star \star \star}$ & 0,000 \\
\hline \multicolumn{5}{|c|}{ Nivel educativo de los padres (ref.: primaria o menos) } \\
\hline Formación Profesional I & $-0,122$ *** & 0,004 & $-0,076^{\star \star \star}$ & 0,004 \\
\hline Secundaria Superior & $-0,168$ *** & 0,004 & $-0,125^{\star \star \star}$ & 0,004 \\
\hline Formación Profesional II & $-0,193 * \star \star$ & 0,005 & $-0,143^{\star \star \star}$ & 0,005 \\
\hline Estudios Universitarios & $-0,313^{* * *}$ & 0,005 & $-0,213^{\star \star *}$ & 0,005 \\
\hline \multicolumn{5}{|c|}{ Situación laboral de los padres en el hogar (ref.: ningún progenitor empleado) } \\
\hline Un padre empleado & $-0,051^{* * *}$ & 0,003 & $-0,045^{\star \star \star}$ & 0,003 \\
\hline Los dos padres empleados & $-0,083$ *** & 0,004 & $-0,081^{* * *}$ & 0,003 \\
\hline Edad & $0,087^{\star \star \star *}$ & 0,001 & 0,064 *** & 0,001 \\
\hline Pseudo R & 0,163 & & 0,141 & \\
\hline Muestra & 95.382 & & 88.603 & \\
\hline
\end{tabular}

* Significatividad al 10\%; ** Significatividad al 5\%; ${ }^{\star \star \star}$ Significatividad al $1 \%$.

Nota: E.M.M.: Efectos marginales medios; E.E.: Error estándar.

Fuente: Elaboración propia a partir de EPA I/2007-IV/2014. 


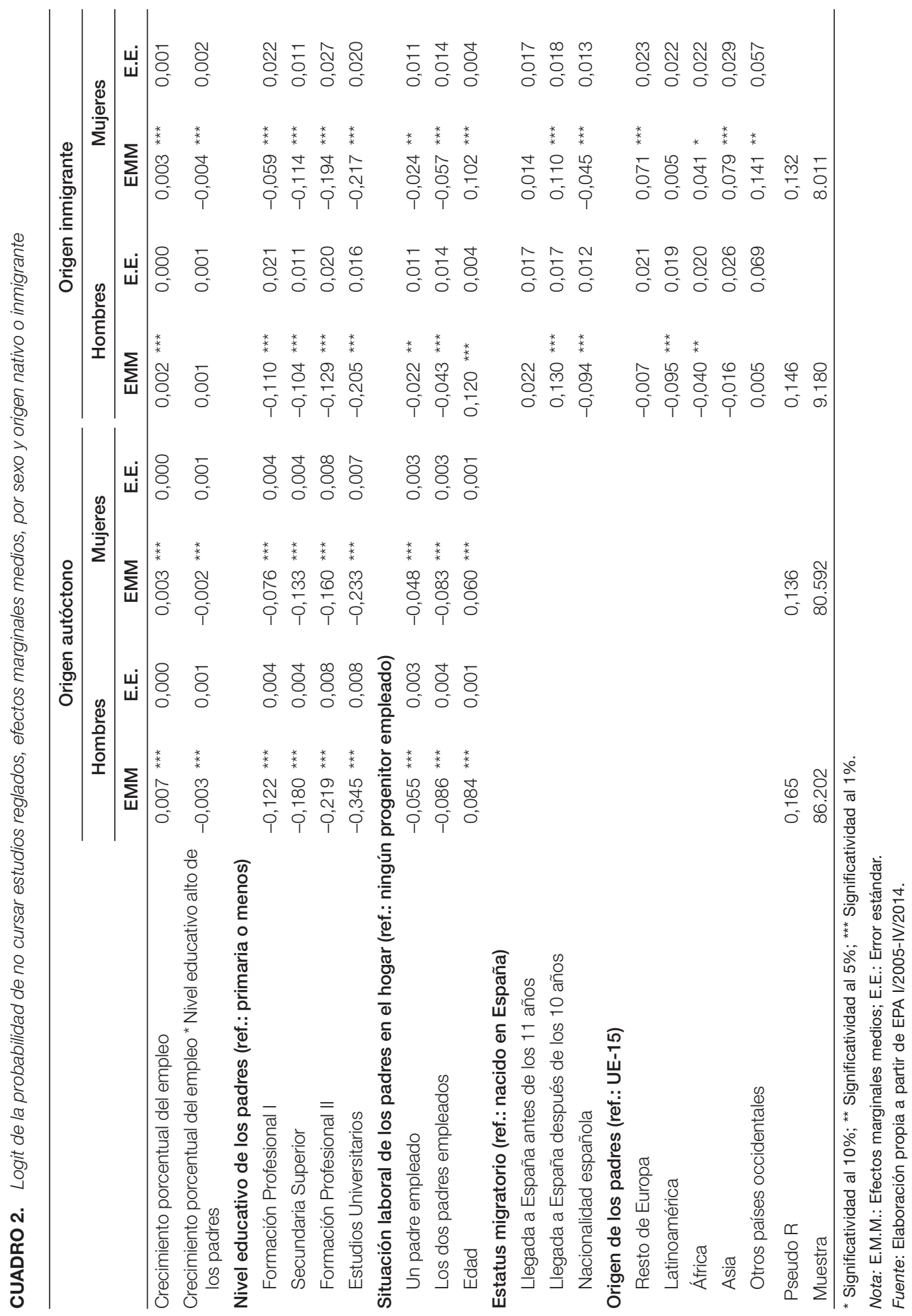


su dedicación a los estudios ante el empeoramiento de las oportunidades de empleo. En tercer lugar, el efecto sustitución no está presente de forma hegemónica. El nivel educativo de los padres no ejerce ningún poder de mediación ante variaciones en la tasa de ocupación para los hombres jóvenes de origen inmigrante. Sin embargo, lo reduce casi a la mitad para los jóvenes de origen autóctono y lo anula para las mujeres de cualquier origen: ante cambios en la tasa de ocupación, las hijas de familias con recursos educativos no modifican su probabilidad de cursar estudios reglados, aunque sí lo hacen las hijas de familias sin estudios superiores.

Por último, en los modelos estimados solo para inmigrantes se han incluido variables específicas del colectivo, como la región de origen de sus padres, el nacimiento en España y, en caso contrario, la edad de llegada (en dos grupos) así como la tenencia de la nacionalidad española. En el caso de los hombres, solo los de origen latinoamericano $y$ africano tienen una probabilidad menor que los de la UE-15 de estar fuera del sistema educativo. Para las mujeres de origen inmigrante, sin embargo, no existe diferencia significativa entre las latinoamericanas y las procedentes de la UE-15, al mismo tiempo que el resto de orígenes considerados presenta una probabilidad significativamente mayor. Además, tener nacionalidad española se relaciona negativamente con la probabilidad de no estudiar. También es importante señalar que no existen diferencias significativas entre los nacidos en España y los que llegaron con diez años o menos, pero sí con los que llegaron con 10 años o más.

\section{Discusión de los Resultados Y CONCLUSIONES}

Los jóvenes que durante la recesión se incorporan al mercado de trabajo deben de afrontar condiciones especialmente difíciles. La literatura sugiere que el paso por largos periodos de desempleo durante la fase inicial en el mercado de trabajo puede tener consecuencias sobre toda su trayectoria laboral (Felgueroso, et al., 2014). Los jóvenes residentes en España se han enfrentado a esa coyuntura económica tras un periodo que desincentivó su continuidad en el sistema educativo. Durante el periodo de expansión económica que terminó en 2007, España se ha diferenciado, junto con otros países del sur de Europa, del patrón de disminución del abandono escolar de la media europea. Este elevado abandono escolar tuvo especial protagonismo entre los hombres debido fundamentalmente a la abundante oferta de puestos de trabajo bien remunerados en el expansivo sector de la construcción. Durante los años de auge económico, además, habían llegado importantes flujos migratorios a España, que habían ocupado en su mayoría los puestos de trabajo de baja cualificación que la economía estaba creando. El aumento de la población activa de origen extranjero trajo consigo también la incorporación a la sociedad española, bien a través de la reagrupación o a través de la reproducción en destino, de la generación de inmigrantes 1.5 y segunda generación. Este grupo de jóvenes presentaba tasas de abandono escolar superiores a las de sus compañeros de origen nativo. La irrupción de la recesión en 2008, que afectó especialmente al sector de la construcción, coincidió con un descenso generalizado del abandono escolar, especialmente el masculino, y también en gran medida el de los jóvenes de origen inmigrante.

Por estas razones supone este un relevante caso de estudio sobre las dinámicas de dedicación a los estudios de los jóvenes de origen inmigrante y los de origen nativo. La literatura sobre inmigración ha puesto de relieve la posible ventaja de los inmigrantes en el sistema educativo en base a la hipótesis del optimismo inmigrante, pero también las posibles dificultades a las que se enfrentan a través de la hipótesis de la aculturación. 
Por su parte, desde las teorías de la estratificación social se ha puesto el foco del análisis en el efecto del origen social sobre distintos aspectos del logro educativo, entre ellos la dedicación a los estudios, y los efectos a largo plazo sobre el bienestar y la desigualdad social. Desde esta óptica se ha estudiado el efecto de una recesión económica sobre la desigualdad en el abandono escolar. Así, se pueden esperar dos mecanismos opuestos según refuercen o suavicen la desigualdad social en la probabilidad de estar fuera del sistema educativo. Mientras que la disminución de las oportunidades laborales de los menos cualificados puede contribuir a la reducción del abandono escolar entre las clases menos acomodadas, a través de un efecto sustitución, el incremento de costes y la necesidad de conseguir ingresos pueden incrementar el abandono escolar de estas clases, a través de un efecto renta. Las diferencias en la dinámica de dedicación a los estudios de los jóvenes de origen inmigrante tendrán su origen, por lo tanto, en dos componentes: el atribuido a su origen inmigrante y el componente de su distinta composición de origen social, por lo general con menos recursos.

Este trabajo se proponía contribuir a la literatura sobre dedicación a los estudios poniendo de relieve, en primer lugar, el sentido en que opera el componente inmigrante ante cambios en el contexto económico. El segundo objetivo de este trabajo consistía en analizar los mecanismos a través de los que operan los recursos educativos paternos en la probabilidad de estar fuera del sistema educativo para los dos grupos de población.

El trabajo empírico realizado pone en evidencia que en la sociedad española los jóvenes de origen inmigrante, una vez controladas otras características sociodemográficas, tienen una mayor probabilidad de estar fuera del sistema educativo que los hijos de autóctonos, de modo que no se encuentra, en este caso, sustento empírico para la hipótesis del optimismo de los inmigrantes. No obstante, esto solo aplica para los descendientes de los inmigrantes que han llegado a España una vez cumplidos los 10 años. La ausencia de diferencias significativas (o el reducido tamaño de estas) en la dedicación a los estudios entre los jóvenes de origen inmigrante nacidos en España o llegados a temprana edad y los hijos de autóctonos supone una buena noticia sobre su integración social.

En segundo lugar, a la luz de los datos se puede afirmar que los jóvenes descendientes de inmigrantes han reaccionado menos ante cambios en su probabilidad de estar empleados que sus pares de origen autóctono. En efecto, a pesar de que la reducción de su distancia con los autóctonos en el porcentaje de jóvenes fuera del sistema educativo disminuyó hasta 2012, esta contracción se hizo en un contexto en el que las condiciones del mercado de trabajo habían empeorado sustancialmente para los jóvenes inmigrantes. En definitiva, las evidencias sugieren que los jóvenes de familias de origen inmigrante se resisten más a aumentar su presencia en las escuelas ante un empeoramiento de las probabilidades de empleo que les afecta especialmente.

Tercero, no se han encontrado evidencias de que en un contexto de deterioro de las condiciones del mercado de trabajo se produzca un efecto renta, de forma tal que las familias con menos recursos educativos sean las que más disminuyan su dedicación a los estudios. Al contrario, sí se encuentra un efecto sustitución, pero que en ningún caso es hegemónico o uniforme entre los cuatro grupos considerados. Entre los hombres jóvenes de origen autóctono, son los de familias con menos recursos educativos los que más reaccionan al cambio de contexto laboral, disminuyendo por lo tanto la desigualdad social dentro del grupo respecto a la dedicación a los estudios cuando empeoran las oportunidades laborales. Asimismo, tanto las mujeres de origen autóctono como las de origen inmigrante de familias 
con más recursos educativos no reaccionan en absoluto a los cambios en la probabilidad de estar empleadas, aunque sí lo hacen las hijas de padres sin estudios superiores, de modo que entre las mujeres el efecto sustitución es máximo. Este efecto, sin embargo, no se da entre los varones de origen inmigrante, entre los que no se observa ningún efecto diferencial del contexto laboral en función de los recursos educativos familiares.

La recesión económica ha tenido efectos adversos muy intensos sobre el bienestar y la cohesión social. Sin embargo, aunque el abandono escolar sigue siendo muy superior a la media europea, la recesión también ha conseguido frenar una dinámica social que podría tener consecuencias negativas sobre el bienestar social a largo plazo. A pesar de ello, los jóvenes de origen inmigrante no han reaccionado tan positivamente como los de origen autóctono al cambio en el contexto económico, perdiendo la oportunidad de convertir sus peores condiciones laborales en un recorte de distancia con los autóctonos en términos de inversión educativa. La recuperación de los porcentajes de jóvenes de origen inmigrante que no cursan estudios reglados en la fase final del periodo analizado sugiere que una recuperación económica podría alargar la brecha entre los jóvenes en cuanto a su dotación de recursos educativos. La investigación futura tendrá que poner de relieve los mecanismos que han frenado un mayor vuelco hacia el sistema educativo por parte de los jóvenes de origen inmigrante.

\section{Bibliografía}

Bernardi, Fabrizio; Garrido, Luis y Miyar-Busto, María (2011). «The Recent Fast Upsurge of Immigrants in Spain and Their Employment Patterns and Occupational Attainment». International Migration, 49(1): 148-187.

Boudon, Raymond (1974). «Education, Opportunity, and Social Inequality: Changing Prospects in Western Society». Disponible en: http://eric.ed. gov/?id=ED091493, acceso 14 de marzo de 2015.
Castro Martín, Teresa y Rosero-Bixby, Luis (2011). «Maternidades y fronteras. La fecundidad de las mujeres inmigrantes en España». Revista Internacional de Sociología, 69(M1): 105-138.

Causa, Orsetta y Jean, Sébastien (2007). «Integration of Immigrants in OECD Countries». Disponible en: http://www.oecd-ilibrary.org/economics/integration-of-immigrants-in-oecd-countries_162367775052

Cebolla-Boado, Héctor (2011). «Primary and Secondary Effects in the Explanation of Disadvantage in Education: The Children of Immigrant Families in France». British Journal of Sociology of Education, 32(3): 407-430.

Cebolla-Boado, Héctor y Martínez de Lizarrondo, Antidio (2015). « ¿Las expectativas educativas de la población inmigrante en Navarra? ¿Optimismo inmigrante o efectos de escuela?». Revista Internacional de Sociología, 73(1): e007.

Cebolla-Boado, Héctor; Miyar-Busto, María y MuñozComet, Jacobo (2015). «Is the Spanish Recession Increasing Inequality? Male Migrant-Native Differences in Educational Returns Against Unemployment». Journal of Ethnic and Migration Studies, 41(5): 710-728.

Cebolla-Boado, Héctor; Radl, Jonas y Salazar, Leire (2015). "Colección Estudios Sociales, 39. Aprendizaje y ciclo vital». Disponible en: http:// obrasocial.lacaixa.es/deployedfiles/obrasocial/ Estaticos/pdf/Estudios_sociales/vol39_es.pdf, acceso 14 de marzo de 2015.

Dolado, Juan J.; Jansen, Marcel; Felgueroso, Florentino; Fuentes, Andrés y Wölfl, Anita (2013). «Youth Labour Market Performance in Spain and its Determinants». Disponible en: http://www.oecd-ilibrary.org/content/workingpaper/5k487n5bfz5cen, acceso 14 de marzo de 2015.

Driscoll, Anne K. (1999). «Risk of High School Dropout among Immigrant and Native Hispanic Youth». International Migration Review, 857875.

Farkas, George (1996). Human Capital or Cultural Capital?: Ethnicity and Poverty Groups in an Urban School District. Disponible en: https://books. google.es/books?hl=es\&lr=\&id=_CmvGsWXwYc C\&oi=fnd\&pg=PR13\&dq=Human+Capital+or+Cu Itural+Capital\%3F+Ethnicity+and+Poverty+Grou ps+in+an+Urban+School+District.\&ots $=B 1 D 74 b$ uONo\&sig=17n_LYtEs_Uc_8WKZDZITCszevY, acceso 14 de marzo de 2015. 
Farley, Reynolds y Alba, Richard (2002). «The New Second Generation in the United States». International Migration Review, 36(3): 669-701.

Felgueroso, Florentino; Gutiérrez-Domènech, María y Jiménez-Martín, Sergi (2014). «Dropout Trends and Educational Reforms: The Role of the LOGSE in Spain». IZA Journal of Labor Policy, 3(1): 1-24.

Garrido Medina, Luis J.; Miyar-Busto, María y MuñozComet, Jacobo (2010). «La dinámica laboral de los inmigrantes en el cambio de fase del ciclo económico". Presupuesto y Gasto Público, 61: 201-221.

Greenman, Emily (2013). «Educational Attitudes, School Peer Context, and the "Immigrant Paradox" in Education". Social Science Research, 42 (3): 698-714.

Haller, William; Portes, Alejandro y Lynch, Scott M. (2011). «Dreams Fulfilled, Dreams Shattered: Determinants of Segmented Assimilation in the Second Generation». Social Forces, 89(3): 733-762.

Harris, Angel L.; Jamison, Kenneth M. y Trujillo, Mónica H. (2008). «Disparities in the Educational Success of Immigrants: An Assessment of the Immigrant Effect for Asians and Latinos». The Annals of the American Academy of Political and Social Science, 620(1): 90-114.

Heckman, James J. y LaFontaine, Paul A. (2010). "The American High School Graduation Rate: Trends and Levels». The Review of Economics and Statistics, 92(2): 244-262.

Hirschman, Charles (2001). «The Educational Enrollment of Immigrant Youth: A Test of the Segmented-Assimilation Hypothesis». Demography, 38(3): 317-336.

Jonsson, Jan O. y Rudolphi, Frida (2011). «Weak Performance-strong Determination: School Achievement and Educational Choice among Children of Immigrants in Sweden». European Sociological Review, 27(4): 487-508.

Kao, Grace y Tienda, Marta (1995). "Optimism and Achievement: The Educational Performance of Immigrant Youth». Social Science Quaterly, 76(1): 1-19.

Kogan, Irena (2006). «Labor Markets and Economic Incorporation among Recent Immigrants in Europe». Social Forces, 85(2): 697-721.

Landale, Nancy S.; Oropesa, R. Salvador y Llanes, Daniel (1998). «Schooling, Work, and Idleness among Mexican and Non-Latino White Adoles- cents». Social Science Research, 27(4): 457-480.

Mood, Carina (2010). «Logistic Regression: Why We Cannot Do What We Think We Can Do, and What We Can Do about It». European Sociological Review, 26(1): 67-82.

Muñoz-Comet, Jacobo (2013). «La salida del desempleo de extranjeros y españoles. Efectos del contexto económico". Revista Española de Investigaciones Sociológicas, 142(1): 45-67.

Perreira, Krista M.; Mullan Harris, Kathleen y Lee, Dohoon (2006). «Making It in America: High School Completion by Immigrant and Native Youth». Demography, 43(3): 511-536.

Pong, Suet-ling; Hao, Lingxin y Gardner, Erica (2005). "The Roles of Parenting Styles and Social Capital in the School Performance of Immigrant Asian and Hispanic Adolescents». Social Science Quarterly, 86(4): 928-950.

Portes, Alejandro y Zhou, Min (1993). «The New Second Generation: Segmented Assimilation and its Variants». The Annals of the American Academy of Political and Social Science, 530(1): 74-96.

Requena, Miguel y Sánchez-Domínguez, María (2011). "Las familias inmigrantes en España». Revista Internacional de Sociología, 69(M1): 79-104.

Rumbaut, Ruben G. (1997). «Assimilation and its Discontents: Between Rhetoric and Reality". International Migration Review, 923-960.

Scarpetta, Stefano; Sonnet, Anne y Manfredi, Thomas (2010). «Rising Youth Unemployment during the Crisis». Disponible en: http://www.oecd-ilibrary. org/social-issues-migration-health/rising-youthunemployment-during-the-crisis_5kmh79zb2mmv-en, acceso 14 de marzo de 2015.

Waldinger, Roger y Feliciano, Cynthia (2004). «Will the New Second Generation Experience "downward Assimilation"? Segmented Assimilation Re-Assessed». Ethnic and Racial Studies, 27(3): 376-402.

White, Michael J. y Glick, Jennifer E. (2009). Achieving Anew: How New Immigrants Do in American Schools, Jobs, and Neighborhoods. Disponible en: https://books.google.es/books?hl=es\&lr=\&id $=x S X 80 Y \mid p w 0 E C \& o i=$ fnd\&pg $=P R 5 \& d q=$ White,+ M.J.,+Glick,+J.E.,+2009.+Achieving+Anew:+Ho $w+N e w+I m m i g r a n t s+D o+i n+A m e r i c a n+S c h o o l s$, +Jobs,+and+Neighborhoods.+Russell+Sage+Fo undation+Publications, + New+York. \&ots $=$ Hf1Pm PPJdL\&sig = wXP1P6RmRJMPCkLZkgbtEAGIOg, acceso 14 de marzo de 2015. 
Xie, Yu y Greenman, Emily (2011). «The Social Context of Assimilation: Testing Implications of Segmented Assimilation Theory". Social Science Research, 40(3): 965-984.
Zhou, Min (1997). «Growing up American: The Challenge Confronting Immigrant Children and Children of Immigrants». Annual Review of Sociology, 63-95.

RECEPCIÓN: 05/10/2015

REVISIÓN: 14/01/2016

APROBACIÓN: 07/04/2016 



\title{
Immigrant-origin Youth Engagement in Education in Spain during the Great Recession
}

\author{
La dedicación a los estudios de los jóvenes de origen inmigrante \\ en España en la Gran Recesión
}

María Miyar-Busto

\section{Key words}

Second Generation Immigrants

- 1.5 Generation

- School Dropout

- Spain

- Immigrant Optimism

Hypothesis

- Acculturation

\section{Palabras clave}

Segunda generación de inmigrantes

- Generaciones 1.5

- Abandono escolar

- España

- Hipótesis del

optimismo del

inmigrante

- Aculturación

\begin{abstract}
This paper analyses the patterns of engagement in education of young people of immigrant origin resident in Spain, using data from the Spanish Labour Force Survey. The results show that the probability that immigrant-origin youth may be outside the educational system is higher than the probability for native youth, but only for those who came to Spain after the age of ten years old. In addition, their reaction to changes in the employment situation has been less intense. Also, parental educational resources slow down the reaction to changes in employment opportunities for the children of native people and daughters of immigrants, decreasing the inequality within each group in contexts of recession, but this is not the case for male young immigrants.
\end{abstract}

\section{Resumen}

En este trabajo se analizan las pautas de dedicación a los estudios de la población joven de origen inmigrante residente en España, a partir de datos de la Encuesta de Población Activa. Los resultados muestran que la probabilidad de estar fuera del sistema educativo de los jóvenes de origen inmigrante es superior a la de los de origen autóctono, aunque solo para los que llegaron a España después de los diez años. Además, su reacción ante cambios en las circunstancias laborales ha sido menos intensa. Asimismo, se encuentra que los recursos educativos paternos frenan la reacción ante cambios en las oportunidades laborales para los hijos de autóctonos y las mujeres hijas de inmigrantes, disminuyendo la desigualdad dentro de cada grupo en contextos de recesión, pero no sucede así para los varones de origen inmigrante.

\section{Citation}

Miyar-Busto, María (2017). "Immigrant-origin Youth Engagement in Education in Spain during the Great Recession". Revista Española de Investigaciones Sociológicas, 157: 123-140. (http://dx.doi.org/10.5477/cis/reis.157.123)

María Miyar-Busto: Departamento de Sociología II (Estructura Social), Universidad Nacional de Educación a Distancia (UNED) | mmiyar@poli.uned.es 


\section{INTRODUCTION ${ }^{1}$}

Abundant theoretical and empirical evidence has been found in the sociology of education to validate the relationship between labour market evolution and the unequal engagement in education of young people from different social backgrounds. The literature on the assimilation of immigrants has analysed the different patterns of engagement in education among immigrant-origin and native children. This article proposes bringing the two literatures together, and analysing the behaviour of each group with respect to early school leaving in the light of changes in the employment situation in Spain.

The study of the engagement in education of second-generation immigrants not only provides relevant information on the progress of the assimilation process in the country of destination, but is also useful for predicting future patterns of economic and social inequality. In addition to the impact that early school leaving has on the labour market, as the population without specific training becomes part of the job-seeking workforce (Scarpetta et al., 2010), it has effects on social welfare, health and citizen participation (Heckman and LaFontaine, 2010).

This article seeks to make three main contributions to the literature on early school leaving. Firstly, a theoretical contribution, by connecting (i) the social stratification theories on the dynamics of social origin in determining educational outcomes with (ii) the literature on the assimilation of second-generation immigrants. Secondly, it aims to enhance the existing empirical knowledge about the integration of the children of immigrants in Spain through

\footnotetext{
1 This study was carried out within the R \& D \& I project entitled "Reducing Structural Unemployment in Spain: Training and Employment, Labour Costs, Migration, Welfare State and Family" (CSO2014-59927-R), funded by the Spanish Ministry of Economy and Competitiveness.
}

the analysis of a key element in their future social integration. The analysis of their engagement in education provides information about the processes of assimilation and the perpetuation of inequality among immigrants and natives across generations. Thirdly, it attempts to provide a better understanding of the long-term effect of the changes in the economic cycle on second-generation immigrants. Data from the Spanish Labour Force Survey (LFS) for the period 2007-2014 will be used to analyse the patterns of non-engagement in education among the children of immigrants and the children of natives.

The article has two objectives. The first is to explore how being of immigrant origin affects the possible change of patterns of engagement in education as the economic cycle changes. And the second is to explore the different role that parents' educational resources play in the education of immigrants' and natives' children, respectively, in this change of dynamics.

There have been numerous contributions to the literature on the effects that an economic recession can have on different aspects of inequality between natives and immigrants, such as the impact on the labour market (Muñoz-Comet, 2013; Garrido et al., 2010) and the returns provided by education (Cebolla-Boado et al., 2015). However, no analyses have been made to date of the effect that a recession may have on the unequal probability of being outside the education system based on immigrant or native origin.

The structure of the paper is as follows. Firstly, a description of the changes in the resident population in Spain over time will be provided, and of the behaviour of the labour market in relation to the patterns of non-engagement in education. Secondly, the main theoretical approaches to the analysis of early school leaving, as well as the study's hypotheses, will be presented. Thirdly, the data, variables, and methodology used in the 
empirical part of the analysis will be detailed. Fourthly, the results of descriptive and multivariate analyses will be described, followed by the conclusions.

\section{YOUTH AT THE INTERSECTION OF THE EDUCATION SYSTEM, THE LABOUR MARKET AND THE ECONOMIC CYCLE IN SPAIN}

In the late 1970s, Spain experienced a decreasing trend in school drop-out rates, which reversed in the mid-1990s due to factors such as the increasing availability of lowskilled jobs and the change in the design of educational pathways (Felgueroso et al., 2014). This trend contrasted with the pattern of decreasing school drop-out rates in the whole of the EU-15. According to Eurostat data from the European Labour Force Surveys, in a year of full economic boom such as 2005 the school drop-out rate in Spain stood at $37 \%$ of men and $25 \%$ of women, compared with $20 \%$ and $15 \%$, respectively, for the whole of the EU-15.

As a result of the severe recession experienced by the Spanish economy, more than three million jobs have been destroyed since 2008. The literature has shown that rising unemployment particularly affected low-skilled young people (Dolado et al., 2013) and the sectors and jobs mainly occupied by immigrants during the boom (Bernardi et al., 2011). This coincided with an increasing importance of education in determining the employment situation during the recession (Cebolla-Boado et al., 2015).

According to data from Eurostat, the school drop-out rate in Spain has been in decline since 2008, especially among men, although it is still higher than among women. In 2014 it affected $26 \%$ of men, compared with $18 \%$ of women. Despite the significant improvement, the figures are still considerably higher than the European average, which also declined during the same period, and in 2014 affected $13 \%$ of men and 10\% of women.

Both during the economic boom and after the change in the economic cycle, immigrant population has created family-building projects, either through reunification or by forming new family units in the country of destination (Castro Martín and Rosero-Bixby, 2011; Requena and Sánchez-Domínguez, 2011). According to Residential Variation Statistics, the percentage of children under 16 years old of the total inflow of foreign-born population remained at around $15 \%$ from 2000 to 2014 . In addition, the percentage of births to foreign mothers exceeded $20 \%$ of all births in 2008. An indicator of the intense process of family building of new residents was the dramatic increase in the foreign registered population under 16 years old living in Spain, which grew from 133,391 in 2000 to 798,601 in 2014 . This is therefore a sizable and growing group, which in the literature on the assimilation of second-generation immigrants (or 1.5 generation) is expected to have different patterns of inclusion into the education system after the age of compulsory schooling.

\section{Determinants of the ENGAGEMENT IN EDUCATION FOR NATIVE CHILDREN AND IMMIGRANTS' CHILDREN}

Two analytical approaches have been used in the literature to explain the determinants of immigrants' engagement in education when faced with changes in the economic environment. The first relates to theories of educational stratification that analyse the determinants of educational attainment among different population groups, and the second approach emphasises the relationship between school enrolment and changes in the economic cycle.

The theories of educational stratification have formulated a classic distinction that in- 
volves the existence of two processes to explain educational attainment: on the one hand, educational outcomes or cognitive skills (primary effects), and on the other hand, educational choices, made on the basis of a cost-benefit structure (also called secondary effects) (Boudon, 1974).

Several recent studies in the sociology of education have provided empirical evidence about the disadvantage that immigrants experience when compared with natives in terms of primary effects, while enjoying an advantage in secondary effects for the cases of Sweden (Jonsson and Rudolphi 2011), France (Cebolla-Boado, 2011) and Navarra (Cebolla-Boado and Martínez de Lizarrondo, 2015). This argument is consistent with the immigrant optimism hypothesis (Kao and Tienda, 1995), which predicted that immigrants' children would outperform their peers, once controlled for the disadvantages they faced, such as poorer language skills and a lower social class position. This greater net engagement in education could be accounted for by a greater emphasis placed by immigrant families on the importance of educational attainment and family values. There is abundant literature that has tried to validate this hypothesis, especially for the North American case (Driscoll, 1999; Harris et al., 2008; Landale et al. 1998; Perreira et al., 2006. Pong et al., 2005; Rumbaut, 1997; White and Glick, 2009).

In contrast to this type of analysis, the studies that have focused on the assimilation of second-generation immigrants have found there to be a net disadvantage in their educational attainment. This disadvantage has been explained by the segmented-assimilation hypothesis, which posits that immigrants have different patterns of social mobility depending on the resources available upon their arrival. Accordingly, while some may have patterns of upward social mobility, others cannot follow that path. Another group may undertake limited assimilation, encouraging educational aspirations for their chil- dren, but restricting their integration into the host culture. This analytical approach has paid special attention to the concept of acculturation that second-generation immigrants may experience (Greenman, 2013). From this perspective, the segmented-assimilation hypothesis implies that secondgeneration immigrants who do not follow upwardly mobility patterns will have lower educational aspirations (Portes and Zhou, 1993). The literature has found conflicting evidence. While Hirschman (2001) found empirical evidence of the existence of acculturation and segmented assimilation in data from the United States, he also showed that its existence depended largely on the origin of immigrants and the age at which they moved to the United States. Haller et al. (2011) also found a pattern of segmented assimilation and downward mobility. However, Farley and Alba (2002), Waldinger and Feliciano (2004) and Xie and Greenman (2011) found no evidence that second-generation immigrants experience downward mobility.

The second analytical approach that is relevant to this paper discusses the unequal impact that a change in the economic cycle has on school drop-out rates based on social origin, as the structure of costs and benefits of continuing to study varies across different population groups. In the light of an external shock such as an economic recession, the literature posits two possible mechanisms, with opposite effects on the extent to which students from different social classes continue to pursue their education. On the one hand, there may be a substitution effect, whereby reduced opportunities in the labour market (given the destruction of low-level occupations) would decrease the opportunity cost for students to further their education and therefore increase the likelihood that young people from disadvantaged social backgrounds would remain in the education system. On the other hand, an income effect may also arise, according to which the decline in household budgets, rising education- 
al costs and a decreasing quality in education reduces the engagement in education among those from the lowest social positions to a greater extent than among those from higher social positions (Cebolla-Boado et al., 2015).

Based on these approaches, three possible sources of differentiation can be deduced in the drop-out patterns of immigrantorigin youth in a context of economic change:

A) Immigrants may show different school drop-out patterns either because they are immersed in acculturation processes, as proposed by the segmented-assimilation hypothesis, or because of their higher social mobility aspirations, as proposed in the immigrant optimism hypothesis. Again, how the immigrant origin component operates depends on which of the two elements works more intensely on the aspirations of the second generation and their families. This immigrant origin component may have consequences on both the probability of not engaging in education and on the ability to react to changes in the economic cycle.

B) The deterioration of job opportunities is usually more extreme among the lowest positions in the labour market, in which immigrants are usually employed (Farkas, 1996; Zhou, 1997), especially in Southern Europe (Cause and Jean 2007; Kogan, 2006). The social origin component would therefore be in operation here. How this component operates would depend on the relative weights of the income effect and the substitution effect.

C) The social origin component may operate differently among the children of immigrants than among native children. Immigrant families in less well-off positions are more likely to lack the necessary public support or social networks to avoid placing another family member in the labour market. Alternatively, the possibly higher value given to educational attainment by these families may have a more positive effect than that among native families of the same social origin. The immigrant origin component operates here as a mediator of the social origin component.

\section{Hypotheses}

This study seeks to test the following two hypotheses and their alternatives on the basis of two research questions:

1) Do immigrants' children react more strongly to changes in the economic environment in their likelihood of not pursuing formal education?

H1a: Immigrants' children increase their engagement in education more than native children in a period of economic recession.

H1b: Immigrants' children increase their engagement in education less than native children in a period of economic recession.

2) Does immigrant origin operate as a mediator of the effect of social origin when faced with periods of economic change?

H2a: The immigrant origin component operates as a mediator that in relative terms improves the reaction of children of immigrants with fewer educational resources in periods of economic change.

H2b: The immigrant origin component operates as a mediator that in relative terms worsens the reaction of children of immigrants with fewer educational resources in periods of economic change.

\section{Data, VARIABles AND METHOdS}

Microdata from the Spanish Labour Force Survey (LFS) was used throughout. This survey provides information on labour market developments and household characteristics. It is a quarterly survey that has been carried 
out in Spain since 1964 and has a sample of some 60,000 households, and a population of 180,000 people. In this analysis, survey data between 2007 and 2014 was used. This period includes the starting point of the recession (2007 was the last year of the boom period in which the employment rate grew) until the year in which the employment rate increased again. The choice of this period means that information about the year of arrival of all young people born abroad is also available? .

The population under analysis was between the ages of 16 (the ending age of compulsory education) and 19 years old. In order to be able to cover information about the social origin in the analysis, the group analysed was restricted to those living in the same household as at least one of their parents. For the 16-19 age group, according to the LFS data, the excluded group was in a minority. The total sample of this group for the period studied was 183,985 young people, of whom 17,191 had foreign-born parents.

The dependent variable for the study was not pursuing any type of formal education. The main independent variables included the immigrant origin of the youth, specified as having both parents born outside Spain (or one of them if they only lived with one). Young immigrants were also classified according to whether they had been born in Spain; and, when they were not Spanish-born, whether they had arrived before or after they were 10 years old. This would take into account the length of residence, which was expected to be a key variable in the social integration of young people. To ease the analysis, the children of mixed couples were excluded, as their results were very similar to those of native children 3 .

\footnotetext{
2 Until 2007 the LFS only included information about years of residence in the country of non-Spanish nationals. From 2007 this variable has also been included for Spanish nationals born outside Spain.

3 Estimations available on demand.
}

Information on parental educational resources was also included (the higher education level of the two parents was considered). The educational level of parents was classified as Lower Secondary or less, Vocational Training 1, Upper Secondary, Vocational Training 2, and University Degree. For some of the descriptive analysis, and for analyses which included an interaction between labour market development and parental education level, this variable was added as a dichotomous variable in which the children of immigrants with upper-level Vocational Training or a University Degree took the value of 1. A variable relating to material household resources was also included, which was specified in terms of the employment status of the parents: whether neither or only one of them was employed, or whether both were employed.

To estimate the effect of the labour market developments on the calculations, an independent variable was included: the percentage change in the percentage of those employed in the total age group (16 to 19) of each sex and migratory origin. This captured the variation in the true probability that the young people in each demographic group had of finding a job.

For estimates restricted to the immigrant population, some variables specific to that group that were expected to have an influence on their engagement in education were included: region of origin of parents (EU-15, Rest of Europe, Latin America, Africa, Asia and other Western countries) and whether or not they had Spanish nationality.

A separate analysis was performed for men and women, given the different patterns of their employment behaviour and their engagement in education. The analysis was also controlled for the age variable.

The methodology for the empirical analysis consisted of two steps. Firstly, a descriptive analysis was conducted of the time pattern of non-engagement in education of 
young people based on their immigration status, sex and parental educational resources. Secondly, a series of logistic regression models were estimated. In the first block of models the probability of not pursuing any type of formal education was estimated for all young people, including an interaction between variation in the employment rate and youth migration background. In the second block of models, estimates were carried out separately for native and immigrant youth, including an interaction between the variation in the employment rate and the educational level of the parents. Estimates for young people of immigrant origin were also supplemented by group-specific variables that could influence their process of social integration.

The tables below show the average marginal effects, which allow for the comparison of coefficients between estimated models for different samples and different variables (Mood, 2010), standard errors and significance levels.

\section{Results}

\section{Descriptive analysis}

Figure 1 shows that the percentage of young people who did not engage in formal education during the period analysed was higher among the children of immigrants than among children of natives, and lower among women of both groups. In 2007, almost a third of native young males and a fifth of native young women were not engaged in any kind of formal education, compared to $48 \%$ of young men and $38 \%$ of young women of immigrant origin.

However, the fact that the four groups evolved differently in terms of their presence

FIGURE 1: Percentage of young people not enrolled in formal education, by sex and native or immigrant origin, 16-19 years old

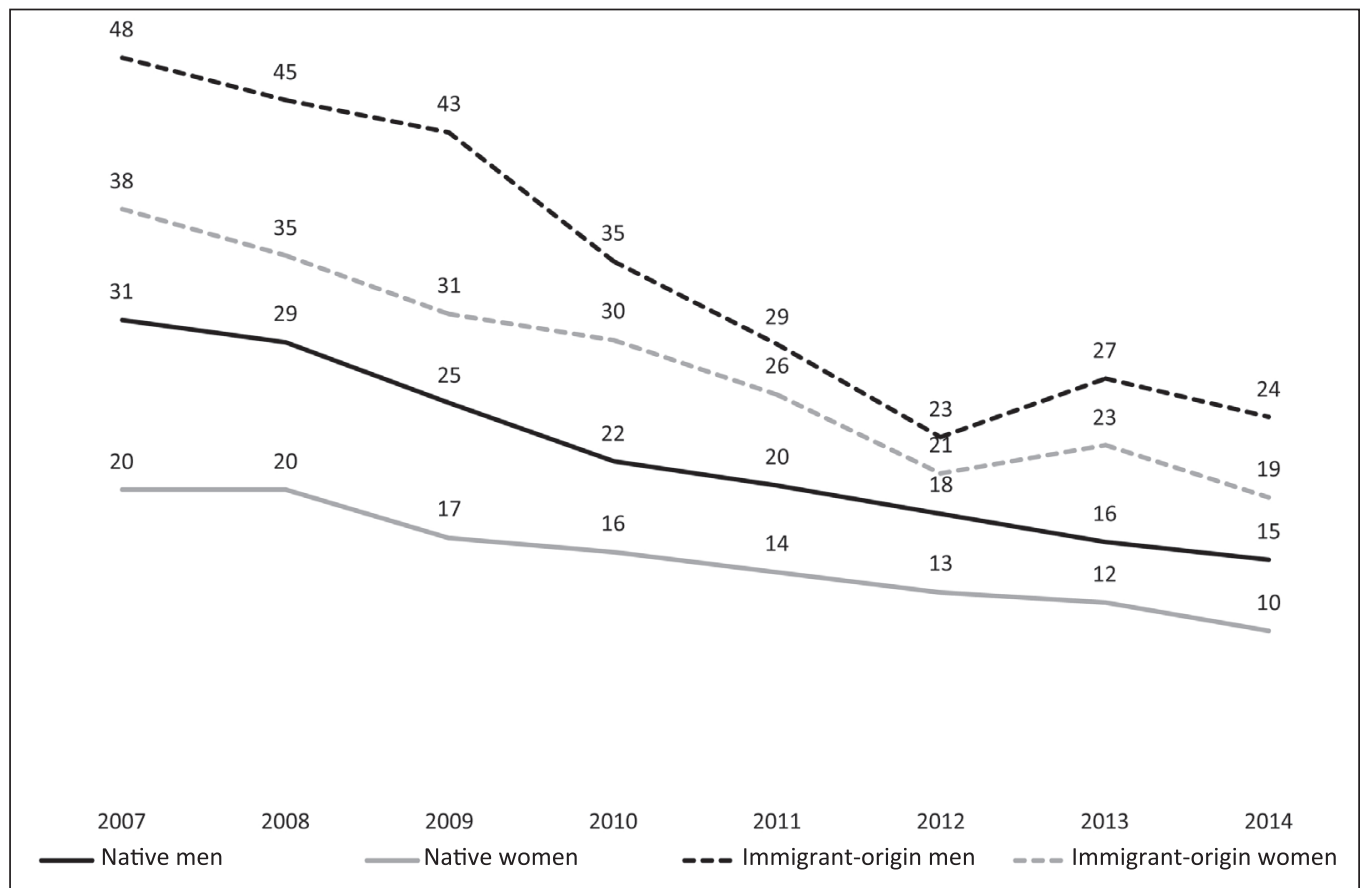

Source: Author's calculations based on the Spanish LFS 2007-2014. 
in the educational system meant that the distance was not constant throughout the period studied. Both native young males and females showed a mild decrease in the percentages of non-students since 2007 , which accelerated from 2008. Moreover, among immigrant-origin young men the reduction in the percentage of non-students did not accelerate until 2009, while among immigrantorigin women the reduction had already been noticeable since 2007.

In addition, since 2009 the contraction in the percentage of young people outside the education system happened faster among children of immigrants than among native children. The distance between the two groups became increasingly small, and reached the lowest level in 2012. From that year onwards, whereas for native young people the previous pattern of decline in the percentage of those who were not engaged in formal education persisted, the trend slowed down for immigrant-origin youth, and their presence in education even increased slightly. In $2014,15 \%$ of native young males were not engaged in formal education, compared with $24 \%$ of immigrant origin. Among women, the corresponding figures were 10 and $19 \%$, respectively.

Figures 2 and 3 show (on a separate basis) how the four groups fared in terms of the percentage of non-students for the children of parents with and without higher education (university education or upper-level vocational training). Three different types of dynamics can be seen. First, the percentage of youth not engaged in formal education was lower among children of parents with higher education for all groups considered by immigrant origin and sex. The average for native young men and women whose parents did not have higher education was $28 \%$ and $19 \%$ respectively for this period, while for the children of parents with higher education it

FIGURE 2: Percentage of young people not enrolled in formal education, by sex and native or immigrant origin, 16 to 19 years old, parents without higher education

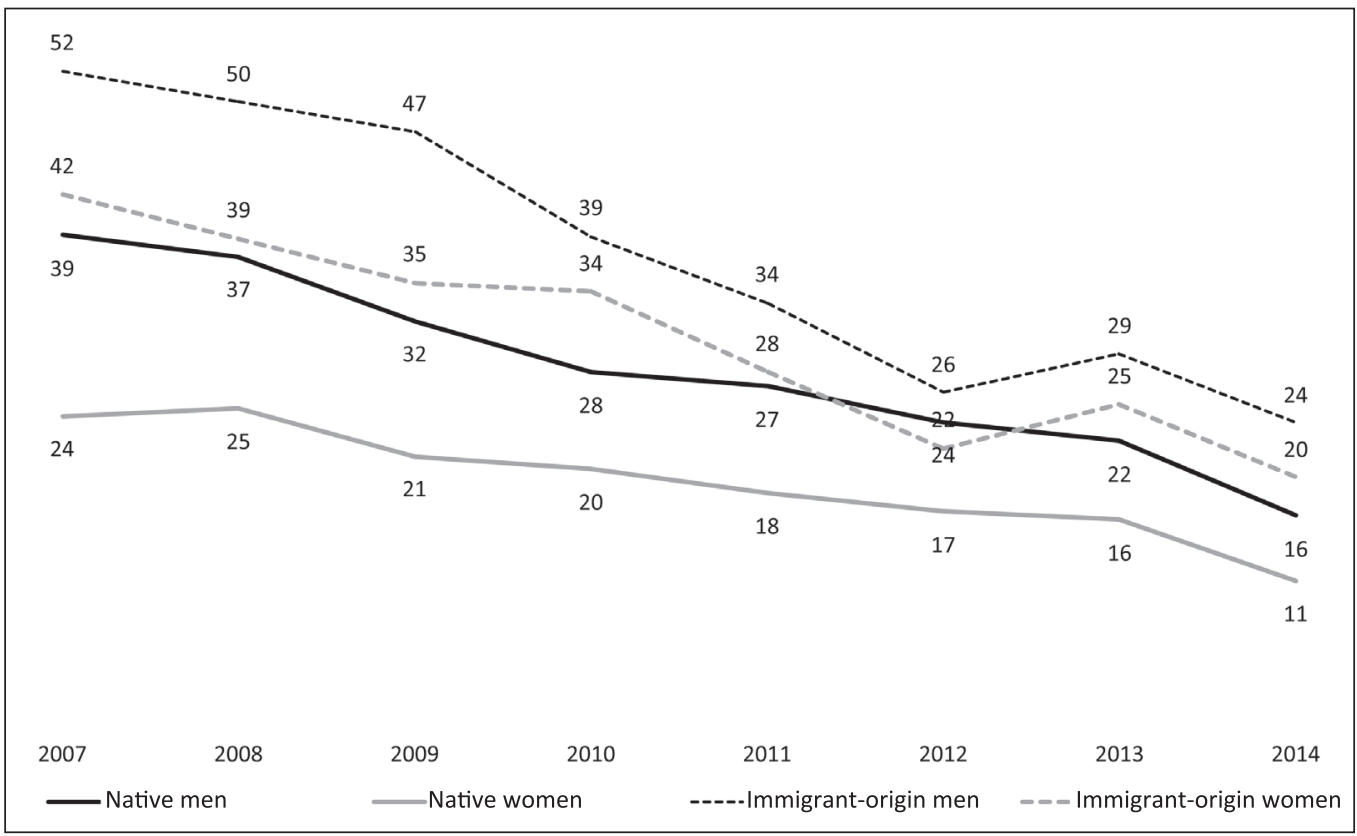

Source: Author's calculations based on the Spanish LFS 2007-2014. 
FIGURE 3: Percentage of young people not enrolled in formal education, by sex and native or immigrant origin, 16 to 19 years old, parents with higher education

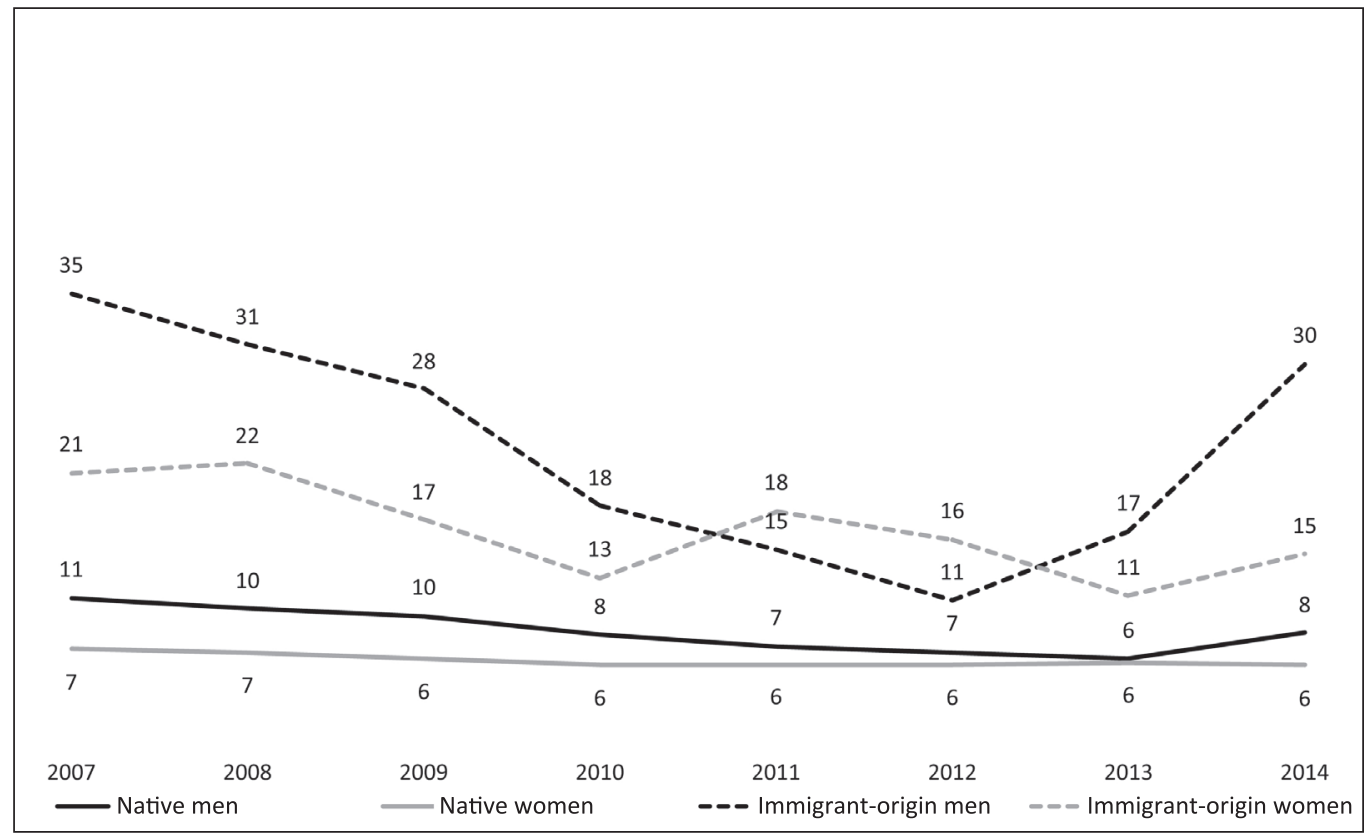

Source: Author's calculations based on the Spanish LFS 2007-2014.

was 9 and 6\%, respectively. Among immigrant-origin youth, the percentages were 38 and $31 \%$ for children of parents without higher education, as opposed to 23 and $17 \%$ for children of parents with higher education.

It should also be noted that the distance depending on immigration status was greater among young people whose parents had greater educational resources than among the children of parents with lower education levels. While in the average for the period studied, the difference related to being of immigrant origin in terms of the percentage of non-students was 33 and $60 \%$ among children of parents without higher education, this distance was $160 \%$ among children of parents with higher education, for both men and women.

Finally, although the two groups by education level fared similarly over time with regard to the distance between children of im- migrant and native origin, the magnitudes differed. While in 2014 the relationship between the percentages of young people from both immigrant and native origins who did not engage in formal education remained similar to pre-crisis levels for children of parents with low education levels, the distance had grown among children with more educated parents, primarily due to differential patterns from 2012 onwards.

\section{Multivariate models}

The results of the multivariate analyses confirmed that, for this age group, being of immigrant origin conditioned the probability of being in the educational system (Table 1). However, among men, only those who came to Spain after the age of ten had a higher probability than the children of native parents of not being involved in formal education (10\% higher). In fact, young men with immi- 
grant parents who were born in Spain had 4 percent lower probability than those of native origin of not studying, while no significant difference was found among those who arrived before age 10. Among women, although those born in Spain of immigrant parents showed no significant difference with respect to the daughters of native parents, those who arrived in Spain before age 10 were 3\% more likely to be outside the education system, while those who arrived at the age of 10 or above were $12 \%$ more likely.

It follows from the above results that changes in the employment rate have a greater effect on men's engagement in education than on women's. For each percentage point change in the employment rate, the probability of not studying increased by $0.6 \%$ for men and $0.3 \%$ for women. In 2012 , the percentage change in the employment rate of native young people was $-9.1 \%$, which would mean, according to the estimates, a reduction of $5.5 \%$ in the probability of being outside the education system. However, young people of immigrant origin saw this effect reduced by $0.5 \%$ per percentage point, which means that their sensitivity to changes in labour market conditions was significantly lower. In the face of a decrease in the employment rate, their presence in the education system increased less than that of native youth. In the case of women, the interaction of occupation changes with the immigrant component was reduced to $0.1 \%$. Therefore, it could be stated that the differ-

TABLE 1. Logit of the probability of not being enrolled in formal education, average marginal effects by sex

\begin{tabular}{|c|c|c|c|c|}
\hline & \multicolumn{2}{|c|}{ Men } & \multicolumn{2}{|c|}{ Women } \\
\hline & A.M.E. & S.E. & A.M.E. & S.E. \\
\hline \multicolumn{5}{|l|}{ Origin (ref.: Native son) } \\
\hline Son of inmigrants born in Spain & $-0.04^{\star * \star}$ & 0.010 & 0.01 & 0.009 \\
\hline Arrived in Spain before turning 11 & -0.01 & 0.007 & $0.03^{* \star *}$ & 0.006 \\
\hline Arrived in Spain after turning 10 & $0.10^{\star \star \star}$ & 0.006 & $0.12^{\star \star \star}$ & 0.005 \\
\hline Employment growth & $0.006^{\star \star \star}$ & 0.000 & $0.003^{* \star *}$ & 0.000 \\
\hline Employment growth * immigrant origin & $-0.005^{* \star \star}$ & 0.000 & 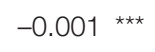 & 0.000 \\
\hline \multicolumn{5}{|c|}{ Parents' educational level (ref.: primary or less) } \\
\hline Vocational Training 1 & $-0.122^{\star \star \star}$ & 0.004 & $-0.076^{\star \star \star}$ & 0.004 \\
\hline Upper Secondary & $-0.168^{\star \star \star}$ & 0.004 & $-0.125^{\star \star \star}$ & 0.004 \\
\hline Vocational Training 2 & $-0.193^{* \star *}$ & 0.005 & $-0.143^{* \star *}$ & 0.005 \\
\hline Higher Education & $-0.313^{* \star \star}$ & 0.005 & $-0.213^{\star \star \star}$ & 0.005 \\
\hline \multicolumn{5}{|c|}{ Parents' employment status in the household (ref.: neither parent in work) } \\
\hline One parent employed & $-0.051^{\star \star \star *}$ & 0.003 & $-0.045^{* * *}$ & 0.003 \\
\hline Both parents employed & $-0.083^{\star \star \star}$ & 0.004 & $-0.081^{\star \star \star}$ & 0.003 \\
\hline Age & $0.087^{\star \star \star}$ & 0.001 & $0.064^{* \star *}$ & 0.001 \\
\hline Pseudo R & 0.163 & & 0.141 & \\
\hline Sample & 95,382 & & 88,603 & \\
\hline
\end{tabular}

*: Significant at $10 \%$; ${ }^{\star *}$ : Significant at $5 \% ;{ }^{* \star *}$ : Significant at $1 \%$.

Note: A.M.E. Average marginal effects; S.E.: Standard errors.

Source: Author's calculations from the Spanish LFS I/2007-IV/2014. 
ence in the level of their reaction with respect to their native peers was lower than in the case of men.

The estimation also confirmed other recurring predictions in the sociological literature on engagement in education, such as the negative influence of the higher educational level of parents on the probability of being outside the education system. The sons and daughters of university educated parents had a $31 \%$ and $21 \%$ lower likelihood, respectively, of being outside the education system than the children of parents educated to lower secondary or lower levels. The effect of the educational resources of parents was therefore $50 \%$ higher for young men than for young women. In addition, engagement in formal education was more closely related to parental education level than to the employment status of parents. A young man with two employed parents was $8 \%$ less likely to be out of formal education than a young person with no employed parents in the household.

The second group of models (Table 2) provides further insights into the different mechanisms that affected the probability of being outside the education system. Separate estimations were performed based on immigrant origin and sex, which included an interaction variable between the educational level of parents and the variation in the employment rate. This covered any possible differences in intensity and direction of the effect of social origin when faced with changes in employment conditions.

It follows from the results of the estimations: firstly, that educational resources play a smaller role in determining the likelihood of young people of immigrant origin not engaging in formal education, especially for men; secondly, that changes in the labour market did not have an income effect on the probability of being outside the education system, since in none of the four cases considered did young people in households with fewer educational resources react by reducing their engagement in education with the worsening of employment opportunities; and thirdly, that the substitution effect was not present in a hegemonic way. The educational level of the parents did not have any mediation power in the face of changes in the employment rate for young men of immigrant origin. However, parental educational level reduced it by almost half for native young people and rendered it non-existent for women of any origin: when changes in the employment rate took place, the probability that the daughters of families with educational resources may engage in formal education did not change, although it did change in the case of daughters of families without higher education.

Finally, in the models estimated only for immigrants, variables specific to this group were included, such as the parental region of origin, whether or not they had been born in Spain, and if not, the age of arrival (in two groups) and whether or not they had Spanish nationality. In the case of men, only those of Latin American and African origin were less likely than those from the EU-15 to be outside the educational system. For women of immigrant origin, however, there was no significant difference between those from Latin America and those from the EU-15, while the other origins considered presented a significantly higher probability. In addition, having Spanish nationality was negatively related to the probability of not studying. It is also important to note that there were no significant differences between those born in Spain and those who came to Spain at the age of 10 years old or under, but some differences were observed when compared with those who arrived at the age of 10 or above.

\section{Discussion OF RESULTS AND CONCLUSIONS}

Young people who enter the labour market during a recession are faced with particularly 


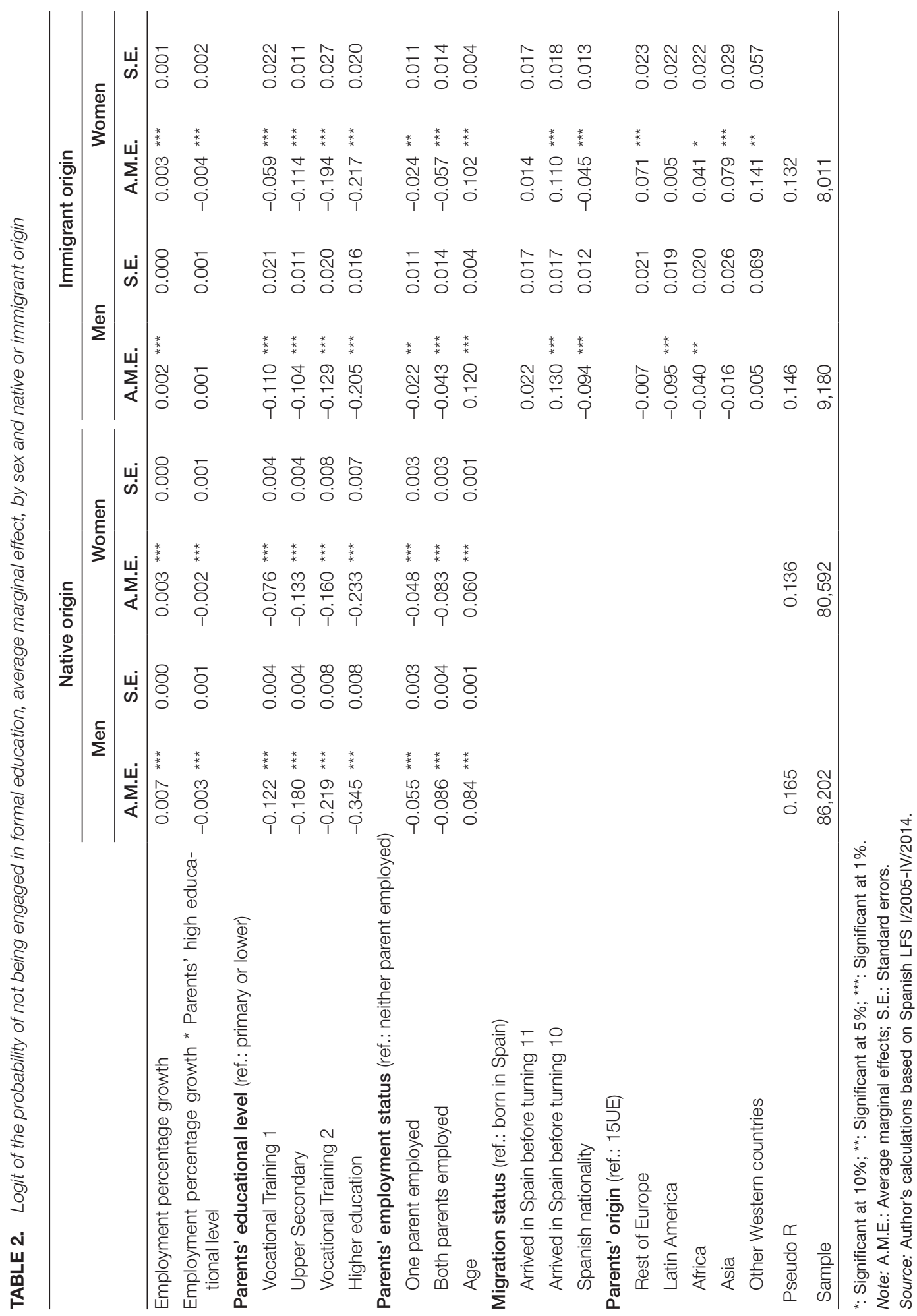


difficult conditions. The literature suggests that experiencing long periods of unemployment during the initial phase of entry into the labour market may have an impact on the person's entire career (Felgueroso et al., 2014). Young residents in Spain have faced this economic situation after a period in which they were discouraged from continuing their education. During the period of economic expansion that ended in 2007 , Spain and the other Southern European countries were not consistent with the European average in terms of a decreasing trend in school drop-out rates. These high school drop-out rates were especially prominent among men, mainly due to the large number of well-paid jobs available in the expanding construction sector. During the years of the economic boom, there were significant migratory flows into Spain, where the newcomers mostly occupied the low-skill jobs created by the economy. As a result of the increase in foreign labour force, 1.5 generation and second-generation immigrants became incorporated into Spanish society, either through reunification or through birth in Spain. This group of young people had higher school drop-out rates than their native peers. The emergence of the recession in 2008, which particularly affected the construction sector, coincided with a general decline in early school leaving, especially for men and of immigrant youth in general.

For the reasons described above, this is a relevant case study on the dynamics of the engagement in education of native and immigrant youth. The migration literature has highlighted the potential advantage of immigrants in the educational system based on the immigrant optimism hypothesis, but also the possible difficulties faced by them, as posed by the acculturation hypothesis. Meanwhile, social stratification theories have focused the analysis on the effect that social origin has on various aspects of educational attainment, including the engagement in education, and the long-term effects on welfare and social inequality. This perspective has been adopted to study the effect of an economic recession on inequality in early school leaving. Two opposing mechanisms can be expected to operate, which either strengthen or weaken social inequality in terms of the probability of being outside the education system. While declining employment opportunities for the less skilled can contribute to the reduction of early school leaving among less affluent classes, through a substitution effect, increased costs and the need to obtain income may increase the drop-out rates of these classes through an income effect. Therefore, the differences in the dynamics of engagement in education of immigrant-origin youth lie in two components: the component attributable to their immigrant origin, and the component related to their different social origin, generally with fewer resources available.

This study was intended to contribute to the literature on engagement in education by highlighting, first, how the immigrant component operates when the economic environment changes. The second objective of this study was to analyse the mechanisms through which parental educational resources affect the probability of the two population groups being outside the educational system.

Firstly, the empirical work undertaken shows that immigrant-origin youth, once other sociodemographic characteristics have been controlled for, are more likely to be outside the educational system than native children in Spanish society. Thus in this case, no empirical support was found for the immigrant optimism hypothesis. However, this only applies to the descendants of immigrants who arrived in Spain after the age of ten years old. The absence-or small number- of significant differences in the engagement in education among young immigrants born in Spain or who arrived at an early age and the children of natives is good news for their social integration. 
Secondly, in light of the data, it can be stated that the young descendants of immigrants have reacted to changes in the probability of being employed less than their native peers. Although the distance between immigrants and natives decreased in the percentage of young people outside the education system until 2012, this contraction took place in a context in which labour market conditions had worsened substantially more for young immigrants. In short, the evidence obtained suggests that young people from immigrant families were more reluctant to increase their presence in education when faced with a lower probability of being employed, that had a particular impact on them.

Thirdly, no evidence has been found that an income effect occurs in the context of deteriorating labour market conditions to the effect that families with fewer educational resources may reduce most their engagement in education. On the contrary, a substitution effect was found, but in no case was it hegemonic or uniform among the four groups considered. In the case of native young men, it was the families with fewer educational resources that reacted most to the changing employment environment, and therefore social inequality was found to decrease within this group during the Recession with regard to their engagement in education. Both native and immigrant-origin women with greater educational resources did not react at all to changes in the probability of being employed, although it did in the case of daughters of families without higher education, so the substitution effect had a major effect on young women. This effect, however, was not seen in immigrant-origin males, among whom no differential effect of the employment context was observed based on family educational resources.

The economic recession has had extremely severe adverse effects on welfare and social cohesion. However, although the early school leaving rate in Spain remains well above the European average, during the recession a social dynamic that could have negative consequences on social welfare in the long term has been halted. Still, young people of immigrant origin have not reacted as positively as natives to the change in the economic environment, and thus have missed an opportunity to turn their worse working conditions into a reduction of the distance between them and native youth in terms of educational investment. The recovery of the percentages of immigrant-origin youth who were not engaged in formal education in the final phase of the review period suggests that an economic recovery could increase the gap among young people in terms of their educational resources. Future research will have to bring to the fore the mechanisms that have slowed down the movement of immigrant-origin youth towards the educational system in Spain.

\section{BiBLIOGRAPHY}

Bernardi, Fabrizio; Garrido, Luis and Miyar-Busto, María (2011). "The Recent Fast Upsurge of Immigrants in Spain and Their Employment Patterns and Occupational Attainment". International Migration, 49(1): 148-187.

Boudon, Raymond (1974). "Education, Opportunity, and Social Inequality: Changing Prospects in Western Society". Available at: http://eric.ed. gov/?id=ED091493, access March 14, 2015.

Castro Martín, Teresa and Rosero-Bixby, Luis (2011). "Maternidades y fronteras. La fecundidad de las mujeres inmigrantes en España". Revista Internacional de Sociología, 69(M1): 105-138.

Causa, Orsetta and Jean, Sébastien (2007). "Integration of Immigrants in OECD Countries". Available at: http://www.oecd-ilibrary.org/economics/integration-of-immigrants-in-oecd-countries_162 367775052

Cebolla-Boado, Héctor (2011). "Primary and Secondary Effects in the Explanation of Disadvantage in Education: The Children of Immigrant Families in France". British Journal of Sociology of Education, 32(3): 407-430.

Cebolla-Boado, Héctor and Martínez de Lizarrondo, Antidio (2015). “¿Las expectativas educativas de 
la población inmigrante en Navarra? ¿Optimismo inmigrante o efectos de escuela?". Revista Internacional de Sociología, 73(1): e007.

Cebolla-Boado, Héctor; Miyar-Busto, María and Muñoz-Comet, Jacobo (2015). "Is the Spanish Recession Increasing Inequality? Male MigrantNative Differences in Educational Returns Against Unemployment". Journal of Ethnic and Migration Studies, 41(5): 710-728.

Cebolla-Boado, Héctor; Radl, Jonas and Salazar, Leire (2015). "Colección Estudios Sociales, 39. Aprendizaje y ciclo vital". Available at: http://obrasocial.lacaixa.es/deployedfiles/obrasocial/Estaticos/pdf/Estudios_sociales/vol39_es.pdf, access March 14, 2015.

Dolado, Juan J.; Jansen, Marcel; Felgueroso, Florentino; Fuentes, Andrés and Wölfl, Anita (2013). "Youth Labour Market Performance in Spain and its Determinants". Available at: http://www.oecdilibrary.org/content/workingpaper/5k487n5bfz5cen, access March 14, 2015.

Driscoll, Anne K. (1999). "Risk of High School Dropout among Immigrant and Native Hispanic Youth". International Migration Review, 857-875.

Farkas, George (1996). Human Capital or Cultural Capital?: Ethnicity and Poverty Groups in an Urban School District. Available at: https://books. google.es/books?hl=es\&lr=\&id=_CmvGsWXwYc C\&oi=fnd\&pg=PR13\&dq=Human + Capital + or $+\mathrm{Cu}$ Itural+Capital\%3F+Ethnicity+and+Poverty+Grou ps+in+an+Urban+School+District.\&ots=B1D74b uONo\&sig=17n_LYtEs_uc_8WKZDZITCszevY, access March 14, 2015.

Farley, Reynolds and Alba, Richard (2002). "The New Second Generation in the United States". International Migration Review, 36(3): 669-701.

Felgueroso, Florentino; Gutiérrez-Domènech, María and Jiménez-Martín, Sergi (2014). "Dropout Trends and Educational Reforms: The Role of the LOGSE in Spain". IZA Journal of Labor Policy, 3(1): 1-24.

Garrido Medina, Luis Joaquín; Miyar-Busto, María and Muñoz Comet, Jacobo (2010). "La dinámica laboral de los inmigrantes en el cambio de fase del ciclo económico". Presupuesto y Gasto Público, 61: 201-221.

Greenman, Emily (2013). "Educational Attitudes, School Peer Context, and the 'Immigrant Paradox' in Education". Social Science Research, 42(3): 698-714.
Haller, William; Portes, Alejandro and Lynch, Scott M. (2011). "Dreams Fulfilled, Dreams Shattered: Determinants of Segmented Assimilation in the Second Generation". Social Forces, 89(3): 733762.

Harris, Angel L.; Jamison, Kenneth M. and Trujillo, Mónica H. (2008). "Disparities in the Educational Success of Immigrants: An Assessment of the Immigrant Effect for Asians and Latinos". The Annals of the American Academy of Political and Social Science, 620(1): 90-114.

Heckman, James J. and LaFontaine, Paul A. (2010). "The American High School Graduation Rate: Trends and Levels". The Review of Economics and Statistics, 92(2): 244-262.

Hirschman, Charles (2001). "The Educational Enrollment of Immigrant Youth: A Test of the Segmented-Assimilation Hypothesis". Demography, 38(3): 317-336.

Jonsson, Jan O. and Rudolphi, Frida (2011). "Weak Performance-strong Determination: School Achievement and Educational Choice among Children of Immigrants in Sweden". European Sociological Review, 27(4): 487-508.

Kao, Grace and Tienda, Marta (1995). "Optimism and Achievement: The Educational Performance of Immigrant Youth". Social Science Quaterly, 76(1): 1-19.

Kogan, Irena (2006). "Labor Markets and Economic Incorporation among Recent Immigrants in Europe". Social Forces, 85(2): 697-721.

Landale, Nancy S.; Oropesa, R. Salvador and Llanes, Daniel (1998). "Schooling, Work, and Idleness among Mexican and Non-Latino White Adolescents". Social Science Research, 27(4): 457-480.

Mood, Carina (2010). "Logistic Regression: Why We Cannot Do What We Think We Can Do, and What We Can Do about It". European Sociological Review, 26(1): 67-82.

Muñoz-Comet, Jacobo (2013). "La salida del desempleo de extranjeros y españoles. Efectos del contexto económico". Revista Española de Investigaciones Sociológicas, 142(1): 45-67.

Perreira, Krista M.; Mullan Harris, Kathleen and Lee, Dohoon (2006). "Making It in America: High School Completion by Immigrant and Native Youth". Demography, 43(3): 511-536.

Pong, Suet-ling; Hao, Lingxin and Gardner, Erica (2005). "The Roles of Parenting Styles and Social 
Capital in the School Performance of Immigrant Asian and Hispanic Adolescents". Social Science Quarterly, 86(4): 928-950.

Portes, Alejandro and Zhou, Min (1993). "The New Second Generation: Segmented Assimilation and its Variants". The Annals of the American Academy of Political and Social Science, 530(1): 7496.

Requena, Miguel and Sánchez-Domínguez, María (2011). "Las familias inmigrantes en España". Revista Internacional de Sociología, 69(M1): 79-104.

Rumbaut, Ruben G. (1997). "Assimilation and its Discontents: Between Rhetoric and Reality". International Migration Review, 923-960.

Scarpetta, Stefano; Sonnet, Anne and Manfredi, Thomas (2010). "Rising Youth Unemployment during the Crisis". Available at: http://www.oecd-ilibrary.org/ social-issues-migration-health/rising-youth-unemployment-during-the-crisis_5kmh79zb2mmv-en, access March 14, 2015.

Waldinger, Roger and Feliciano, Cynthia (2004). "Will the New Second Generation Experience "down- ward Assimilation"? Segmented Assimilation ReAssessed". Ethnic and Racial Studies, 27(3): 376402.

White, Michael J. and Glick, Jennifer E. (2009). Achieving Anew: How New Immigrants Do in American Schools, Jobs, and Neighborhoods. Available at: https://books.google.es/books?hl= es\&l$r=\& i d=x S X 8 O Y \mid p w 0 E C \& o i=f n d \& p g=P R 5 \& d q$ =White,+M.J.,+Glick,+J.E.,+2009.+Achieving+An ew:+How+New+Immigrants+Do+in+American+S chools,+Jobs, +and+Neighborhoods.+Russell+S age+Foundation+Publications,+New+York.\&ots= Hf1PmPPJdL\&sig=wxP1P6RmRJMPCkLZkgbtEAGIOg, access March 14, 2015.

Xie, Yu and Greenman, Emily (2011). "The Social Context of Assimilation: Testing Implications of Segmented Assimilation Theory". Social Science Research, 40(3): 965-984.

Zhou, Min (1997). "Growing up American: The Challenge Confronting Immigrant Children and Children of Immigrants". Annual Review of Sociology, 63-95.

RECEPTION: October 5, 2015

REVIEW: January 14, 2016

ACCEPTANCE: April 7, 2016 